\title{
Linear polarization of a group of symbiotic systems ${ }^{\star}$
}

\author{
E. Brandi ${ }^{1,2}$, L.G. García ${ }^{1}$, V. Piirola ${ }^{3}$, F. Scaltriti ${ }^{4}$, and C. Quiroga ${ }^{1,5}$ \\ 1 Facultad de Ciencias Astronómicas y Geofísicas, Universidad Nacional de La Plata, 1900 La Plata, Argentina \\ 2 Member of the Carrera del Investigador Científico, Comisión de Investigaciones Científicas de la Provincia de Buenos Aires \\ (CIC), Argentina \\ 3 Tuorla Observatory, University of Turku, Finland \\ 4 Osservatorio Astronomico di Torino, Pino Torinese, Italy \\ ${ }^{5}$ Fellow of the Consejo Nacional de Investigaciones Científicas y Técnicas (CONICET), Argentina
}

Received February 21; accepted March 23, 2000

\begin{abstract}
We report linear polarization measurements of a set of symbiotic stars, made at several epochs during the period 1994-1998. Evidence of intrinsic polarization is looked for from the wavelength dependence of the polarization degree and position angle in $U B V R I$ bands. The results have also been analysed to search for temporal variability of polarization. Several objects have shown a polarization spectrum different from that produced by interstellar dust grains and/or polarimetric variations on time scales as short as several days or months, indicating the presence of polarization component of circumstellar origin.
\end{abstract}

Key words: stars: binaries: symbiotic — stars: binaries eclipsing — stars: variable stars — polarization

\section{Introduction}

Symbiotic stars are interacting binary systems in which a cool giant transfers material to a hot companion, responsible for ionization of the gas surrounding the system. Since symbiotic stars are generally embedded in gaseous and dusty envelopes, scattering in the circumbinary nebulae is a likely source for the polarization. Linear polarization of the stellar radiation is, in general, an indication of deviation from spherical symmetry. Therefore, it is to be

Send offprint requests to: E. Brandi

e-mail: ebrandi@fcaglp.unlp.edu.ar

* Based on observations taken at Complejo Astronómico El Leoncito (CASLEO), operated under an agreement between the Consejo Nacional de Investigaciones Científicas y Técnicas de la República Argentina, the Secretaría de Ciencia y Tecnología de la Nación and the National Universities of La Plata, Córdoba and San Juan. expected that the symbiotic stars should possess a significant degree of polarization, since most of them present extended asymmetric atmospheres, circumbinary envelopes and/or dusty circumstellar envelopes, disks, etc. The polarization should be variable with time according to the activity of the star or to the orbital motion. The study of this variability should give a better insight into the envelope structures or their binary nature. Serkowski (1970) reported the first polarimetric study of symbiotic stars. Linear polarization observations of the three symbiotics R Aqr, AG Peg and RS Oph, showed that the measured polarization was intrinsic and variable with time in all those objects. Schulte-Ladbeck, Magalhães (1987) found that 9 out of $23(39 \%)$ of the observed symbiotic stars to be intrinsically polarized. Schulte-Ladbeck et al. (1990) (hereafter SLAMS) found evidence for the presence of intrinsic polarization, by means of the wavelength dependence and/or time variability, in 21 out of 39 objects $(54 \%)$. In order to learn more about the polarizing sources, SLAMS searched correlations between the linear polarization and other properties of the symbiotic systems. Due to circumstellar scattering around the late-type component, they expected to find correlations with IR types S, D and D' and spectral types of the cool component. There are $48 \%$ S-type objects showing intrinsic polarization, $88 \%$ D-type polarized objects and 2 polarized objects out of 4 D'-type objects studied. A higher probability to find polarized D-type symbiotic persists in the larger sample.

Later spectral types would favor, through larger mass loss, the presence of polarization but SLAMS found that the number of polarized symbiotic stars correlated with the spectral type of the cool component is not clear.

Piirola (1988a) made an extensive multicolor polarimetry of $\mathrm{CH}$ Cyg. He found drastic changes in the wavelength dependence of the polarization on a time scale of months and years and small variations in the course of days and weeks. He proposed that the main mechanism 
Table 1. List of objects

\begin{tabular}{|c|c|c|c|c|}
\hline Object & $\begin{array}{c}\text { Cool } \\
\text { Component }\end{array}$ & $I R$-Туре & $\begin{array}{r}\text { Orbit } \\
P(\text { days })\end{array}$ & $\begin{array}{l}\text { al Period } \\
\text { References }\end{array}$ \\
\hline V748 Cen $\left(^{*}\right)$ & M4 & $\mathrm{S}$ & 566.5 & $(1)$ \\
\hline Hen 1103 & M0 & $\mathrm{S}$ & & \\
\hline KXTrA & M6 & $\mathrm{S}$ & & \\
\hline CL Sco & M & $\mathrm{S}$ & 624.7 & (2) \\
\hline $\operatorname{AR~Pav~}(*)$ & M3.7 & $\mathrm{S}$ & 604.5 & (3) \\
\hline FN Sgr & M4 & $\mathrm{S}$ & & \\
\hline Hen 1761 & M5 & $\mathrm{S}$ & & \\
\hline RR Tel & M5 & $\mathrm{D}$ & & \\
\hline $\mathrm{CD}-43^{\circ} 14304$ & K5-M0 & $\mathrm{S}$ yellow & 1448 & (4) \\
\hline AG Peg & M2 & $\mathrm{S}$ & 816.5 & (5) \\
\hline
\end{tabular}

Notes:

(*) eclipsing binary.

(1) van Genderen et al. (1974).

(2) Kenyon (1986).

(3) Bruch et al. (1994).

(4) Schmid et al. (1998) (circular solution).

(5) Fernie (1985).

is scattering by dust particles. The variations were interpreted in terms of formation or growth of dust particles and changes in the density, geometry and particle size distribution of the circumstellar envelope. Very small particles produce polarization increasing steeply toward short wavelength in agreement with the Rayleigh law $\left(P \sim \lambda^{-4}\right)$ and larger dust particles produce a flat wavelength dependence with a drop in the ultraviolet polarization. Possible connections of polarization variations to the orbital motion were suggested by Piirola.

The purpose of this paper is to present new polarization measurements of ten symbiotic stars; to analyze the wavelength behavior of both the percentage of polarization and the position angle; to detect temporal variations and to fit our results with the proposed polarizing mechanisms that could be working in symbiotic stars. In our sample of 10 observed stars, 4 have no previous polarization data (V748 Cen, Hen 1103, CL Sco and FN Sgr); 3 were considered intrinsically polarized (Hen 1761, RR Tel and AG Peg) and 3 represent unpolarized objects or else bordeline cases (KX TrA, AR Pav and CD -43 ${ }^{\circ} 14304$ ). The majority of the stars in our sample are S-type symbiotics; only one, RR Tel is a D-type symbiotic with a Mira variable as cool component and CD $-43^{\circ} 14304$ is considered an S-type yellow symbiotic.

\section{Observations}

The observations were obtained during the interval May 1994 - June 1998 with the five channel photopolarimeter of Turin Observatory, attached at the 2.15-m telescope of CASLEO (San Juan, Argentina). The instrument allows simultaneous measurements of polarization in the five bands $U B V R I$, following the design principles by Piirola $(1973,1988 b)$. The combination of the dichroic beam splitters and the filters cemented to the field lenses of the dichroic filter set matches closely the standard $U B V R I$ system. The final response of the five photomultipliers corresponds to equivalent wavelengths $0.36,0.43$, $0.53,0.65$ and $0.86 \mu \mathrm{m}$.

During each observing run a set of zero and high polarized standards stars were observed to determine both the instrumental polarization and the coordinate transformation into the equatorial system respectively. The net polarization of telescope and instrument, typically $\sim 0.01$ percent, was subtracted from all the data.

\section{Results}

We have obtained polarimetric data for 10 symbiotic systems whose main characteristics are included in Table 1. The results of our observations are presented in the Table 2 where we give for each object, in the different epochs, the average polarization in the $U B V R I$ bands, in terms of the weighted mean values of the Stokes parameters PX and PY, the corresponding degree of linear polarization in percent $P(\%)$, the position angle in degrees PA, and the corresponding mean errors. The number of observations, $N$, is also indicated. Weighting according to the inverse square of the estimated error for each observation is applied. The estimated error is taken either from the least-square fit of the double cosine curves to the eight integrations in the different positions of the $\lambda / 2$ plate, or from the photon statistics, whichever is greater.

The multicolour polarization data were used to determine the wavelength dependence of the degree of polarization and the position angle. In order to investigate whether the observed polarization is purely interstellar, or a significant contribution due to intrinsic polarization exists, we compared the observed spectral distributions of the polarization with that given by the Serkowski's law for the wavelength dependence of the interstellar polarization (Serkowski et al. 1975).

All the symbiotic stars were observed in different epochs and several of them show changes in the wavelength dependence of the polarization on time scales of days or months. Plots of the measured polarization vs. wavelength are presented for each star at the different epochs.

\subsection{Results on individual objects}

\section{V748 Centauri}

V748 Cen has been classified as a $\mathrm{R}$ CrB variable, with a photographic amplitude of about $2 \mathrm{mag}$ and a period 
Table 2. Weighted mean values

\begin{tabular}{|c|c|c|c|c|c|c|c|c|}
\hline $\begin{array}{l}\text { Star/Date/JD } \\
2440000+\end{array}$ & Band & $P X(\%)$ & $P Y(\%)$ & $P(\%)$ & $\mathrm{ME}$ & $\mathrm{PA}$ & $\mathrm{ME}$ & $\mathrm{N}$ \\
\hline \multicolumn{9}{|l|}{ V748 Cen } \\
\hline July 1995 & $\bar{U}$ & .23 & -1.59 & 1.60 & .18 & 139.1 & 3.2 & 19 \\
\hline $9906.54 / 07.58 /$ & $B$ & -.30 & -1.62 & 1.64 & .16 & 129.8 & 2.8 & 19 \\
\hline \multirow[t]{3}{*}{$08.58 / 09.54$} & $V$ & -.10 & -1.13 & 1.14 & .14 & 132.5 & 3.4 & 19 \\
\hline & $R$ & -.28 & -.45 & .53 & .09 & 119.2 & 4.7 & 19 \\
\hline & $I$ & -.26 & -.11 & .29 & .09 & 101.2 & 8.9 & 19 \\
\hline March 1996 & $U$ & .18 & -2.62 & 2.63 & .20 & 137.0 & 2.2 & 4 \\
\hline \multirow[t]{4}{*}{10162.78} & $B$ & .24 & -2.83 & 2.84 & .39 & 137.4 & 3.9 & 4 \\
\hline & $V$ & -.17 & -1.06 & 1.08 & .10 & 130.4 & 2.6 & 4 \\
\hline & $R$ & -.43 & -.85 & .96 & .10 & 121.6 & 2.9 & 4 \\
\hline & $I$ & -.39 & -1.29 & 1.35 & .27 & 126.5 & 5.6 & 4 \\
\hline August 1997 & $U$ & -.50 & -2.04 & 2.10 & .85 & 128.1 & 11.1 & 15 \\
\hline \multirow{4}{*}{$10684.52 / 86.49$} & $B$ & -.30 & -1.53 & 1.56 & .20 & 129.5 & 3.7 & 18 \\
\hline & $V$ & -.22 & -.73 & .76 & .12 & 126.6 & 4.3 & 18 \\
\hline & $R$ & -.28 & -.57 & .64 & .12 & 122.0 & 5.3 & 18 \\
\hline & $I$ & -.22 & -.40 & .46 & .15 & 120.5 & 9.3 & 18 \\
\hline April 1998 & $U$ & -.83 & -1.92 & 2.10 & .12 & 123.3 & 1.7 & 29 \\
\hline $10921.80 / 24.80 /$ & $B$ & -.92 & -1.86 & 2.07 & .14 & 121.8 & 2.0 & 29 \\
\hline \multirow{3}{*}{$27.76 / 28.72$} & $V$ & -.48 & -.64 & .80 & .05 & 116.8 & 1.9 & 29 \\
\hline & $R$ & -.52 & -.64 & .83 & .06 & 115.4 & 2.0 & 29 \\
\hline & $I$ & -.41 & -.27 & .49 & .05 & 106.4 & 3.1 & 29 \\
\hline \multicolumn{9}{|l|}{ Hen 1103} \\
\hline \multirow{5}{*}{$\begin{array}{l}\text { July } 1995 \\
9907.61 / 08.60 / \\
09.57\end{array}$} & $\bar{U}$ & .89 & -1.07 & 1.39 & .62 & -25.1 & $\overline{12.0}$ & 18 \\
\hline & $B$ & .19 & .56 & .59 & .46 & 35.5 & 18.9 & 18 \\
\hline & V & .99 & 1.70 & 1.96 & .44 & 29.9 & 6.3 & 18 \\
\hline & $R$ & .28 & 2.45 & 2.47 & .34 & 41.8 & 3.9 & 13 \\
\hline & $I$ & .22 & 2.06 & 2.08 & .24 & 42.0 & 3.2 & 13 \\
\hline \multirow{5}{*}{$\begin{array}{l}\text { March } 1996 \\
10162.82\end{array}$} & $U$ & 1.40 & 2.72 & 3.06 & .15 & 31.3 & 1.4 & 4 \\
\hline & $B$ & 1.49 & 3.47 & 3.78 & .22 & 33.4 & 1.6 & 4 \\
\hline & $V$ & .79 & 2.59 & 2.70 & .49 & 36.5 & 5.1 & 4 \\
\hline & $R$ & .74 & 3.09 & 3.18 & .44 & 38.3 & 3.9 & 4 \\
\hline & $I$ & .42 & 2.38 & 2.41 & .47 & 40.0 & 5.5 & 4 \\
\hline \multirow{5}{*}{$\begin{array}{l}\text { August } 1997 \\
10684.54 / 87.53\end{array}$} & $U$ & .60 & 1.69 & 1.80 & .08 & 35.2 & 1.3 & 16 \\
\hline & $B$ & .50 & 1.99 & 2.05 & .16 & 38.0 & 2.3 & 16 \\
\hline & $V$ & .36 & 1.71 & 1.75 & .23 & 39.0 & 3.8 & 16 \\
\hline & $R$ & .49 & 1.69 & 1.76 & .24 & 36.9 & 3.9 & 16 \\
\hline & $I$ & .31 & 1.93 & 1.95 & .26 & 40.5 & 3.8 & 16 \\
\hline \multirow{5}{*}{$\begin{array}{l}\text { April } 1998 \\
10922.81 / 28.76\end{array}$} & $U$ & 1.26 & 1.99 & 2.36 & .13 & 28.8 & 1.6 & 18 \\
\hline & $B$ & 1.13 & 2.25 & 2.52 & .09 & 31.6 & 1.0 & 18 \\
\hline & $V$ & .86 & 2.12 & 2.29 & .07 & 33.9 & .9 & 18 \\
\hline & $R$ & .83 & 2.17 & 2.32 & .06 & 34.5 & .8 & 18 \\
\hline & $I$ & .59 & 1.85 & 1.94 & .08 & 36.1 & 1.2 & 18 \\
\hline
\end{tabular}

of 566.5 days (van Genderen et al. 1974). Optical and infrared photometry confirm that V748 Cen is an eclipsing binary and the orbital period is the same as the photographic one. During the eclipse the system becomes much redder, indicating the presence of an M4 giant as the occulting star. Outside the eclipse rapid brightness variations were observed by van Genderen. The presence of a shell absorption spectrum and of emission lines as well as several evident peculiarities in the light curve indicate a system in which shells and/or gas streams are present. Such gas stream is expected to flow from the M giant to the hot component through the inner Lagrangian point. It means that the hot component is seen through a dense region of the gas stream just before eclipse. $\mathrm{H} \alpha$ and other lower Balmer lines are in emission with a strong central absorption component, characteristic to disk-like emission features. 
Table 2. continued

\begin{tabular}{|c|c|c|c|c|c|c|c|c|}
\hline $\begin{array}{l}\text { Star/Date/JD } \\
2440000+\end{array}$ & Band & $P X(\%)$ & $P Y(\%)$ & $P(\%)$ & $\mathrm{ME}$ & $\mathrm{PA}$ & $\mathrm{ME}$ & $\mathrm{N}$ \\
\hline \multicolumn{9}{|l|}{ KX TrA } \\
\hline May 1994 & $\bar{U}$ & .86 & .92 & 1.26 & .14 & 23.5 & 3.2 & 12 \\
\hline \multirow{4}{*}{$9503.71 / 06.69$} & $B$ & .94 & 1.01 & 1.38 & .10 & 23.5 & 2.1 & 12 \\
\hline & $V$ & .99 & 1.36 & 1.68 & .10 & 26.9 & 1.8 & 12 \\
\hline & $R$ & .98 & 1.10 & 1.48 & .07 & 24.2 & 1.3 & 12 \\
\hline & $I$ & 1.01 & .95 & 1.39 & .13 & 21.7 & 2.7 & 12 \\
\hline July 1995 & $U$ & .82 & .90 & 1.22 & .27 & 23.9 & 6.3 & 28 \\
\hline $9904.55 / 05.63 /$ & $B$ & 1.09 & 1.38 & 1.76 & .24 & 25.8 & 3.9 & 28 \\
\hline \multirow[t]{3}{*}{$06.61 / 08.65$} & $V$ & 1.02 & .91 & 1.37 & .14 & 20.8 & 3.0 & 28 \\
\hline & $R$ & .89 & .75 & 1.16 & .10 & 20.1 & 2.5 & 28 \\
\hline & $I$ & .51 & .69 & .86 & .08 & 26.6 & 2.8 & 28 \\
\hline March 1996 & $U$ & .98 & .90 & 1.33 & .15 & 21.3 & 3.3 & 4 \\
\hline \multirow[t]{4}{*}{10162.86} & $B$ & 1.42 & .75 & 1.61 & .23 & 14.0 & 4.0 & 4 \\
\hline & $V$ & .97 & .93 & 1.34 & .04 & 21.9 & .8 & 4 \\
\hline & $R$ & 1.07 & 1.05 & 1.50 & .04 & 22.2 & .7 & 4 \\
\hline & $I$ & .63 & .67 & .92 & .05 & 23.4 & 1.6 & 4 \\
\hline August 1997 & $U$ & .82 & .60 & 1.02 & .12 & 18.1 & 3.4 & 16 \\
\hline \multirow{4}{*}{$10686.57 / 88.49$} & $B$ & 1.04 & .82 & 1.33 & .18 & 19.2 & 3.9 & 16 \\
\hline & $V$ & .85 & .72 & 1.12 & .08 & 20.2 & 2.1 & 16 \\
\hline & $R$ & 1.04 & .76 & 1.29 & .07 & 18.1 & 1.6 & 16 \\
\hline & $I$ & .71 & .62 & .95 & .10 & 20.6 & 3.0 & 16 \\
\hline April 1998 & $U$ & 1.09 & .70 & 1.29 & .17 & 16.3 & 3.7 & 16 \\
\hline \multirow[t]{4}{*}{$10921.87 / 28.80$} & $B$ & 1.12 & 1.00 & 1.51 & .07 & 20.9 & 1.4 & 16 \\
\hline & V & 1.08 & 1.00 & 1.47 & .04 & 21.4 & .9 & 16 \\
\hline & $R$ & 1.04 & 1.08 & 1.50 & .03 & 22.9 & .6 & 16 \\
\hline & $I$ & .84 & 1.00 & 1.30 & .04 & 24.9 & .9 & 16 \\
\hline \multicolumn{9}{|l|}{ CL Sco } \\
\hline 6-11 July 1995 & $\bar{U}$ & 1.19 & 1.46 & 1.89 & .24 & 25.4 & 3.6 & 32 \\
\hline 9904.59/05.68/ & $B$ & 1.09 & 1.03 & 1.50 & .23 & 21.8 & 4.3 & 32 \\
\hline 9906.63/07.65/ & $V$ & .54 & .92 & 1.07 & .18 & 29.7 & 4.8 & 32 \\
\hline \multirow[t]{2}{*}{ 9908.66/09.65 } & $R$ & .36 & .68 & .77 & .15 & 31.1 & 5.7 & 32 \\
\hline & $I$ & .01 & 1.27 & 1.27 & .13 & 44.8 & 2.9 & 32 \\
\hline \multirow{5}{*}{$\begin{array}{l}27 \text { July } 1995 \\
9925.59\end{array}$} & $U$ & -.68 & 1.82 & 1.94 & .20 & 55.2 & 3.0 & 8 \\
\hline & $B$ & -.64 & 1.49 & 1.62 & .47 & 56.6 & 8.1 & 8 \\
\hline & V & -.01 & .83 & .83 & .25 & 45.3 & 8.3 & 8 \\
\hline & $R$ & -.02 & 1.43 & 1.43 & .12 & 45.5 & 2.5 & 8 \\
\hline & $I$ & .28 & 1.01 & 1.05 & .24 & 37.3 & 6.4 & 8 \\
\hline \multirow{5}{*}{$\begin{array}{l}\text { March } 1996 \\
10162.88\end{array}$} & $U$ & .27 & 2.40 & 2.42 & .09 & 41.8 & 1.1 & 4 \\
\hline & $B$ & .42 & 2.97 & 3.00 & .12 & 40.9 & 1.1 & 4 \\
\hline & $V$ & .22 & 2.10 & 2.11 & .04 & 42.0 & .5 & 4 \\
\hline & $R$ & .33 & 2.10 & 2.12 & .03 & 40.5 & .5 & 4 \\
\hline & $I$ & -.19 & 1.61 & 1.62 & .06 & 48.4 & 1.0 & 4 \\
\hline \multirow{5}{*}{$\begin{array}{l}\text { August } 1997 \\
10687.61\end{array}$} & $U$ & .10 & .70 & .71 & .35 & 40.9 & 13.0 & 8 \\
\hline & $B$ & 1.03 & 1.04 & 1.46 & .12 & 22.5 & 2.3 & 8 \\
\hline & $V$ & .46 & 1.12 & 1.21 & .17 & 33.9 & 4.0 & 8 \\
\hline & $R$ & .22 & 1.41 & 1.43 & .17 & 40.7 & 3.5 & 8 \\
\hline & $I$ & -.05 & 1.10 & 1.10 & .15 & 46.3 & 3.9 & 8 \\
\hline \multirow{5}{*}{$\begin{array}{l}\text { April } 1998 \\
10922.84 / 24.83 / \\
28.84\end{array}$} & $U$ & .17 & -.02 & .17 & .08 & -2.9 & 12.5 & 20 \\
\hline & $B$ & .32 & .02 & .33 & .08 & 2.1 & 6.9 & 20 \\
\hline & $V$ & .12 & .80 & .81 & .03 & 40.8 & 1.0 & 20 \\
\hline & $R$ & .09 & .87 & .87 & .02 & 42.0 & .8 & 20 \\
\hline & $I$ & .03 & .99 & .99 & .03 & 44.2 & 1.0 & 20 \\
\hline
\end{tabular}


Table 2. continued

\begin{tabular}{|c|c|c|c|c|c|c|c|c|}
\hline $\begin{array}{l}\text { Star/Date/JD } \\
2440000+\end{array}$ & Band & $P X(\%)$ & $P Y(\%)$ & $P(\%)$ & $\mathrm{ME}$ & $\mathrm{PA}$ & $\mathrm{ME}$ & $\mathrm{N}$ \\
\hline \multicolumn{9}{|l|}{ AR Pav } \\
\hline July 1995 & $U$ & .21 & 1.35 & 1.37 & .16 & 40.6 & 3.4 & 16 \\
\hline \multirow{4}{*}{$9904.73 / 06.76$} & $B$ & .87 & 1.68 & 1.89 & .19 & 31.4 & 2.9 & 16 \\
\hline & $V$ & .55 & 1.52 & 1.62 & .14 & 35.1 & 2.5 & 16 \\
\hline & $R$ & .76 & .83 & 1.12 & .15 & 23.7 & 3.9 & 16 \\
\hline & $I$ & .61 & .50 & .79 & .11 & 19.6 & 3.9 & 16 \\
\hline July 1996 & $U$ & -1.32 & .38 & 1.38 & .11 & 82.0 & 2.3 & 6 \\
\hline \multirow{4}{*}{10276.73} & $B$ & -1.28 & .68 & 1.45 & .08 & 76.1 & 1.5 & 6 \\
\hline & $V$ & -.87 & .66 & 1.10 & .22 & 71.6 & 5.6 & 6 \\
\hline & $R$ & -.38 & .52 & .64 & .26 & 63.1 & 11.0 & 6 \\
\hline & $I$ & -.42 & .51 & .66 & .22 & 64.9 & 9.1 & 6 \\
\hline July 1997 & $U$ & -.30 & .23 & .38 & .08 & 71.1 & 5.8 & 18 \\
\hline \multirow{4}{*}{$\begin{array}{l}10633.68 / 34.70 / \\
36.68\end{array}$} & $B$ & .25 & -.25 & .36 & .07 & 157.5 & 5.3 & 18 \\
\hline & $V$ & .60 & -.14 & .62 & .03 & 173.2 & 1.3 & 18 \\
\hline & $R$ & .64 & -.14 & .66 & .03 & 173.9 & 1.4 & 18 \\
\hline & $I$ & .67 & -.05 & .67 & .04 & 178.0 & 1.8 & 18 \\
\hline August 1997 & $U$ & .04 & .70 & .70 & .17 & 43.3 & 6.8 & 8 \\
\hline \multirow[t]{4}{*}{10687.68} & $B$ & .57 & .12 & .59 & .06 & 185.8 & 3.0 & 8 \\
\hline & $V$ & .75 & .05 & .75 & .04 & 181.8 & 1.4 & 8 \\
\hline & $R$ & .79 & .04 & .79 & .02 & 181.6 & .9 & 8 \\
\hline & $I$ & .67 & -.04 & .67 & .03 & 178.4 & 1.4 & 8 \\
\hline April 1998 & $U$ & -1.06 & .13 & 1.07 & .06 & 86.5 & 1.5 & 8 \\
\hline \multirow{4}{*}{10924.86} & $B$ & -.81 & .08 & .81 & .04 & 87.3 & 1.6 & 8 \\
\hline & $V$ & -.14 & .09 & .17 & .02 & 73.0 & 3.7 & 8 \\
\hline & $R$ & -.04 & .12 & .12 & .03 & 55.0 & 6.0 & 8 \\
\hline & $I$ & -.02 & .15 & .15 & .04 & 48.1 & 7.7 & 8 \\
\hline June 1998 & $U$ & -1.00 & -.04 & 1.00 & .04 & 91.1 & 1.1 & 6 \\
\hline \multirow[t]{4}{*}{10985.67} & $B$ & -.40 & -.31 & .51 & .04 & 108.8 & 2.2 & 6 \\
\hline & V & .22 & -.06 & .22 & .02 & -7.1 & 2.4 & 6 \\
\hline & $R$ & .26 & -.01 & .26 & .02 & -0.5 & 1.7 & 6 \\
\hline & $I$ & .39 & .02 & .39 & .03 & 1.5 & 2.0 & 6 \\
\hline \multicolumn{9}{|l|}{ FN Sgr } \\
\hline May 1994 & $\bar{U}$ & .81 & -.03 & .81 & .09 & -1.1 & 3.3 & 8 \\
\hline \multirow[t]{4}{*}{9505.84} & $B$ & 1.22 & .13 & 1.23 & .10 & 2.9 & 2.3 & 8 \\
\hline & $V$ & .96 & .05 & .96 & .08 & 1.4 & 2.5 & 8 \\
\hline & $R$ & .44 & .02 & .45 & .07 & 1.4 & 4.5 & 8 \\
\hline & $I$ & .33 & .04 & .33 & .08 & 3.9 & 6.8 & 8 \\
\hline 9 July 1995 & $U$ & 2.82 & -.60 & 2.89 & 1.11 & -6.0 & 10.5 & 4 \\
\hline \multirow[t]{4}{*}{9907.76} & $B$ & 2.36 & -.75 & 2.48 & .47 & -8.8 & 5.4 & 4 \\
\hline & V & 1.33 & -.55 & 1.44 & .55 & -11.2 & 10.4 & 4 \\
\hline & $R$ & .63 & 1.37 & 1.51 & .62 & 32.7 & 11.3 & 4 \\
\hline & $I$ & .50 & 1.19 & 1.29 & .47 & 33.7 & 10.1 & 4 \\
\hline 27 July 1995 & $U$ & -.11 & .58 & .59 & .20 & 50.5 & 9.4 & 8 \\
\hline \multirow[t]{4}{*}{9925.64} & $B$ & .24 & .23 & .33 & .17 & 22.2 & 13.5 & 8 \\
\hline & V & .14 & .08 & .16 & .08 & 14.9 & 12.9 & 8 \\
\hline & $R$ & .27 & -.02 & .27 & .07 & -1.8 & 6.9 & 8 \\
\hline & $I$ & .14 & -.06 & .15 & .09 & -11.2 & 14.9 & 8 \\
\hline April 1998 & $U$ & .47 & -.57 & .74 & .07 & -25.2 & 2.8 & 8 \\
\hline \multirow[t]{4}{*}{10924.88} & $B$ & 1.12 & -.21 & 1.14 & .06 & -5.3 & 1.6 & 8 \\
\hline & $V$ & .78 & .12 & .79 & .03 & 4.3 & 1.0 & 8 \\
\hline & $R$ & .76 & .09 & .76 & .02 & 3.4 & .9 & 8 \\
\hline & $I$ & .63 & .16 & .65 & .05 & 7.2 & 2.2 & 8 \\
\hline
\end{tabular}


Table 2. continued

\begin{tabular}{|c|c|c|c|c|c|c|c|c|}
\hline $\begin{array}{l}\text { Star/Date/JD } \\
2440000+\end{array}$ & Band & $P X(\%)$ & $P Y(\%)$ & $P(\%)$ & $\mathrm{ME}$ & $\mathrm{PA}$ & $\mathrm{ME}$ & $\mathrm{N}$ \\
\hline \multicolumn{9}{|l|}{ Hen 1761} \\
\hline October 1994 & $U$ & 1.08 & -.16 & 1.10 & .10 & -4.3 & 2.5 & 32 \\
\hline $9639.57 / 40.58 /$ & $B$ & 1.06 & -.11 & 1.06 & .08 & -3.1 & 2.2 & 32 \\
\hline \multirow[t]{3}{*}{$41.56 / 42.55$} & $V$ & .93 & .23 & .96 & .04 & 7.0 & 1.3 & 32 \\
\hline & $R$ & .91 & .26 & .95 & .02 & 7.9 & .6 & 32 \\
\hline & $I$ & .80 & .20 & .82 & .02 & 7.0 & .7 & 32 \\
\hline 6-9 July 1995 & $U$ & .76 & .48 & .90 & .20 & 16.3 & 6.2 & 32 \\
\hline $9904.78 / 05.83 /$ & $B$ & .84 & .48 & .96 & .12 & 15.0 & 3.4 & 32 \\
\hline \multirow[t]{3}{*}{$06.80 / 07.84$} & $V$ & .99 & .44 & 1.08 & .09 & 11.9 & 2.5 & 32 \\
\hline & $R$ & .72 & .26 & .77 & .08 & 9.9 & 3.0 & 32 \\
\hline & $I$ & .76 & .23 & .79 & .06 & 8.4 & 2.3 & 32 \\
\hline 24 July 1995 & $U$ & .17 & .00 & .17 & .22 & 0.0 & 26.1 & 4 \\
\hline \multirow[t]{4}{*}{9922.77} & $B$ & .43 & .67 & .80 & .17 & 28.5 & 6.0 & 4 \\
\hline & $V$ & .67 & .10 & .67 & .02 & 4.3 & .9 & 4 \\
\hline & $R$ & .80 & .22 & .83 & .02 & 7.6 & .8 & 4 \\
\hline & $I$ & .74 & .09 & .74 & .04 & 3.4 & 1.4 & 4 \\
\hline \multirow{5}{*}{$\begin{array}{l}\text { September } 1995 \\
9990.51\end{array}$} & $U$ & 1.13 & .62 & 1.29 & .17 & 14.3 & 3.7 & 8 \\
\hline & $B$ & .98 & .34 & 1.04 & .09 & 9.6 & 2.5 & 8 \\
\hline & $V$ & .86 & .09 & .86 & .02 & 3.1 & .6 & 8 \\
\hline & $R$ & .98 & .21 & 1.00 & .02 & 6.0 & .6 & 8 \\
\hline & $I$ & .75 & .26 & .80 & .02 & 9.5 & .7 & 8 \\
\hline \multirow{5}{*}{$\begin{array}{l}\text { August } 1997 \\
10684.67 / 87.71 / \\
88.74\end{array}$} & $U$ & .79 & .38 & .87 & .06 & 12.8 & 2.1 & 20 \\
\hline & $B$ & .86 & .34 & .92 & .05 & 10.7 & 1.6 & 20 \\
\hline & V & .82 & .30 & .87 & .02 & 10.1 & .5 & 20 \\
\hline & $R$ & .84 & .32 & .90 & .02 & 10.6 & .5 & 20 \\
\hline & $I$ & .73 & .28 & .78 & .02 & 10.6 & .7 & 20 \\
\hline \multirow{5}{*}{$\begin{array}{l}\text { April } 1998 \\
10927.89\end{array}$} & $U$ & .73 & .31 & .80 & .16 & 11.4 & 5.6 & 16 \\
\hline & $B$ & .75 & .18 & .77 & .12 & 6.8 & 4.6 & 16 \\
\hline & $V$ & .90 & .29 & .95 & .02 & 9.0 & .8 & 16 \\
\hline & $R$ & .85 & .24 & .88 & .03 & 7.9 & 1.0 & 16 \\
\hline & $I$ & .79 & .25 & .83 & .03 & 8.9 & 1.2 & 16 \\
\hline \multicolumn{9}{|l|}{ RR Tel } \\
\hline \multirow{5}{*}{$\begin{array}{l}\text { October } 1994 \\
9640.57 / 41.54 / \\
42.53\end{array}$} & $\bar{U}$ & .64 & -.33 & .72 & .04 & 17.3 & $\overline{1.4}$ & 12 \\
\hline & $B$ & .64 & -.34 & .72 & .05 & 166.1 & 2.1 & 12 \\
\hline & $V$ & .58 & -.24 & .62 & .08 & 168.5 & 3.6 & 12 \\
\hline & $R$ & .57 & -.04 & .57 & .02 & 178.2 & 1.2 & 12 \\
\hline & $I$ & .06 & -.11 & .12 & .04 & 149.1 & 9.8 & 12 \\
\hline \multirow{5}{*}{$\begin{array}{l}\text { July } 1995 \\
9907.86 / 09.84\end{array}$} & $U$ & 1.13 & -.21 & 1.15 & .31 & 174.8 & 7.5 & 6 \\
\hline & $B$ & .74 & .34 & .82 & .25 & 12.4 & 8.5 & 6 \\
\hline & $V$ & .53 & .12 & .55 & .20 & 6.4 & 9.8 & 6 \\
\hline & $R$ & .61 & .02 & .61 & .20 & .9 & 8.9 & 6 \\
\hline & $I$ & .24 & -.15 & .28 & .14 & 163.9 & 13.1 & 6 \\
\hline \multirow{5}{*}{$\begin{array}{l}\text { August } 1997 \\
10684.76 / 86.73 / \\
88.75\end{array}$} & $U$ & .40 & -.10 & .41 & .07 & 172.8 & 4.5 & 21 \\
\hline & $B$ & .34 & -.18 & .38 & .09 & 166.0 & 6.6 & 21 \\
\hline & $V$ & .25 & .10 & .27 & .04 & 10.8 & 3.9 & 21 \\
\hline & $R$ & .39 & .04 & .39 & .03 & 2.7 & 2.1 & 21 \\
\hline & $I$ & .15 & .26 & .30 & .05 & 30.3 & 4.6 & 21 \\
\hline \multirow{5}{*}{$\begin{array}{l}\text { April } 1998 \\
10928.87\end{array}$} & $U$ & .42 & -.29 & .51 & .06 & 162.7 & 3.4 & 8 \\
\hline & $B$ & .42 & -.27 & .50 & .04 & 163.6 & 2.4 & 8 \\
\hline & $V$ & .54 & .08 & .55 & .04 & 4.2 & 2.1 & 8 \\
\hline & $R$ & .58 & -.02 & .58 & .02 & 179.1 & 1.1 & 8 \\
\hline & $I$ & .47 & .61 & .77 & .08 & 26.1 & 2.8 & 8 \\
\hline
\end{tabular}


Table 2. continued

\begin{tabular}{|c|c|c|c|c|c|c|c|c|}
\hline $\begin{array}{l}\text { Star/Date/JD } \\
2440000+\end{array}$ & Band & $P X(\%)$ & $P Y(\%)$ & $P(\%)$ & $\mathrm{ME}$ & $\overline{\mathrm{PA}}$ & $\mathrm{ME}$ & $\mathrm{N}$ \\
\hline \multicolumn{9}{|l|}{ 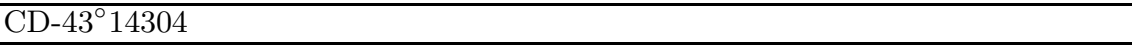 } \\
\hline October 1994 & $U$ & .43 & -.34 & .55 & .14 & 160.8 & 7.1 & 22 \\
\hline $9638.71 / 39.58 /$ & $B$ & .49 & -.21 & .54 & .60 & 168.3 & 24.1 & 23 \\
\hline $40.63 / 41.58 /$ & $V$ & .41 & -.08 & .42 & .17 & 174.7 & 11.0 & 23 \\
\hline \multirow[t]{2}{*}{42.56} & $R$ & .47 & .05 & .47 & .68 & 3.2 & 27.7 & 23 \\
\hline & $I$ & .39 & .02 & .39 & .04 & 1.7 & 2.5 & 23 \\
\hline July 1995 & $U$ & .90 & .19 & .92 & .38 & 6.1 & 11.2 & 30 \\
\hline $9904.81 / 05.85 /$ & $B$ & .91 & .03 & .91 & .30 & 1.0 & 9.2 & 30 \\
\hline 06.83/08.80/ & $V$ & .53 & -.10 & .54 & .18 & 174.8 & 9.4 & 30 \\
\hline \multirow[t]{2}{*}{09.78} & $R$ & .49 & -.16 & .51 & .13 & 171.1 & 7.2 & 30 \\
\hline & $I$ & .27 & -.15 & .31 & .10 & 165.6 & 8.9 & 30 \\
\hline September 1995 & $U$ & .46 & -.29 & .54 & .17 & 163.7 & 8.5 & 8 \\
\hline \multirow[t]{4}{*}{$9992.66 / 93.63$} & $B$ & .47 & -.12 & .48 & .11 & 172.9 & 6.5 & 8 \\
\hline & $V$ & .33 & -.26 & .42 & .03 & 160.7 & 2.0 & 8 \\
\hline & $R$ & .38 & -.21 & .44 & .02 & 165.5 & 1.4 & 8 \\
\hline & $I$ & .23 & -.27 & .35 & .04 & 155.2 & 3.2 & 8 \\
\hline August 1997 & $U$ & .29 & -.22 & .37 & .11 & 161.7 & 8.6 & 8 \\
\hline \multirow[t]{4}{*}{10686.78} & $B$ & .44 & -.30 & .53 & .09 & 162.9 & 4.6 & 8 \\
\hline & $V$ & .39 & -.34 & .51 & .02 & 159.4 & 1.2 & 8 \\
\hline & $R$ & .33 & -.38 & .50 & .02 & 155.5 & 1.0 & 8 \\
\hline & $I$ & .23 & -.31 & .38 & .06 & 153.3 & 4.3 & 8 \\
\hline April 1998 & $U$ & .69 & .86 & 1.11 & .42 & 25.6 & 10.5 & 8 \\
\hline \multirow[t]{4}{*}{10928.90} & $B$ & .33 & -.76 & .83 & .30 & 146.7 & 10.0 & 8 \\
\hline & $V$ & .32 & -.10 & .34 & .09 & 171.0 & 7.8 & 8 \\
\hline & $R$ & .45 & -.07 & .45 & .04 & 175.6 & 2.8 & 8 \\
\hline & $I$ & .15 & .02 & .15 & .12 & 3.0 & 19.7 & 8 \\
\hline \multicolumn{9}{|l|}{ AG Peg } \\
\hline \multirow{5}{*}{$\begin{array}{l}\text { October } 1994 \\
9640.64 / 41.61 / \\
42.60\end{array}$} & $U$ & .18 & -.31 & .36 & .18 & 150.2 & 13.1 & 12 \\
\hline & $B$ & .07 & -.07 & .10 & .08 & 157.8 & 18.7 & 12 \\
\hline & $V$ & .06 & -.17 & .18 & .08 & 144.0 & 12.1 & 12 \\
\hline & $R$ & -.03 & -.00 & .03 & .04 & 93.2 & 24.0 & 12 \\
\hline & $I$ & .04 & -.04 & .06 & .05 & 157.6 & 19.6 & 12 \\
\hline \multirow{5}{*}{$\begin{array}{l}\text { July } 1995 \\
9904.86 / 06.85 / \\
07.90 / 08.86 / \\
09.87\end{array}$} & $U$ & .31 & .28 & .42 & .37 & 20.8 & 20.7 & 18 \\
\hline & $B$ & .06 & .01 & .06 & .27 & 5.7 & 39.2 & 18 \\
\hline & $V$ & -.28 & -.02 & .28 & .21 & 91.8 & 18.2 & 18 \\
\hline & $R$ & -.60 & .09 & .61 & .28 & 85.6 & 12.1 & 18 \\
\hline & $I$ & -.35 & .13 & .37 & .15 & 79.5 & 11.0 & 18 \\
\hline \multirow{5}{*}{$\begin{array}{l}\text { September } 1995 \\
9992.68 / 93.66\end{array}$} & $U$ & -.15 & .29 & .33 & .22 & 58.4 & 16.9 & 8 \\
\hline & $B$ & .02 & -.14 & .14 & .13 & 139.8 & 21.0 & 8 \\
\hline & $V$ & -.09 & -.18 & .20 & .03 & 122.2 & 3.7 & 8 \\
\hline & $R$ & -.03 & -.12 & .12 & .02 & 128.5 & 4.9 & 8 \\
\hline & $I$ & -.13 & -.08 & .15 & .04 & 105.5 & 7.0 & 8 \\
\hline \multirow{5}{*}{$\begin{array}{l}\text { August } 1997 \\
10684.73 / 86.71\end{array}$} & $U$ & -.01 & -.06 & .06 & .08 & 132.4 & 26.6 & 12 \\
\hline & $B$ & -.12 & -.06 & .14 & .07 & 103.2 & 13.3 & 12 \\
\hline & $V$ & -.02 & -.10 & .11 & .04 & 129.4 & 9.8 & 12 \\
\hline & $R$ & -.12 & -.08 & .14 & .02 & 106.3 & 5.0 & 12 \\
\hline & $I$ & -.09 & -.08 & .12 & .04 & 110.5 & 10.4 & 12 \\
\hline
\end{tabular}

V748 Cen was observed in July 1995, March 1996, August 1997 and April 1998 and there are no previous polarimetric observations of this object. Figure 1 shows the weighted mean values of the degree of polarization and the position angle as a function of wavelength and for the different observational runs. The amount of polarization changes with wavelength and a sharp increase of the degree of polarization to the short wavelengths was 

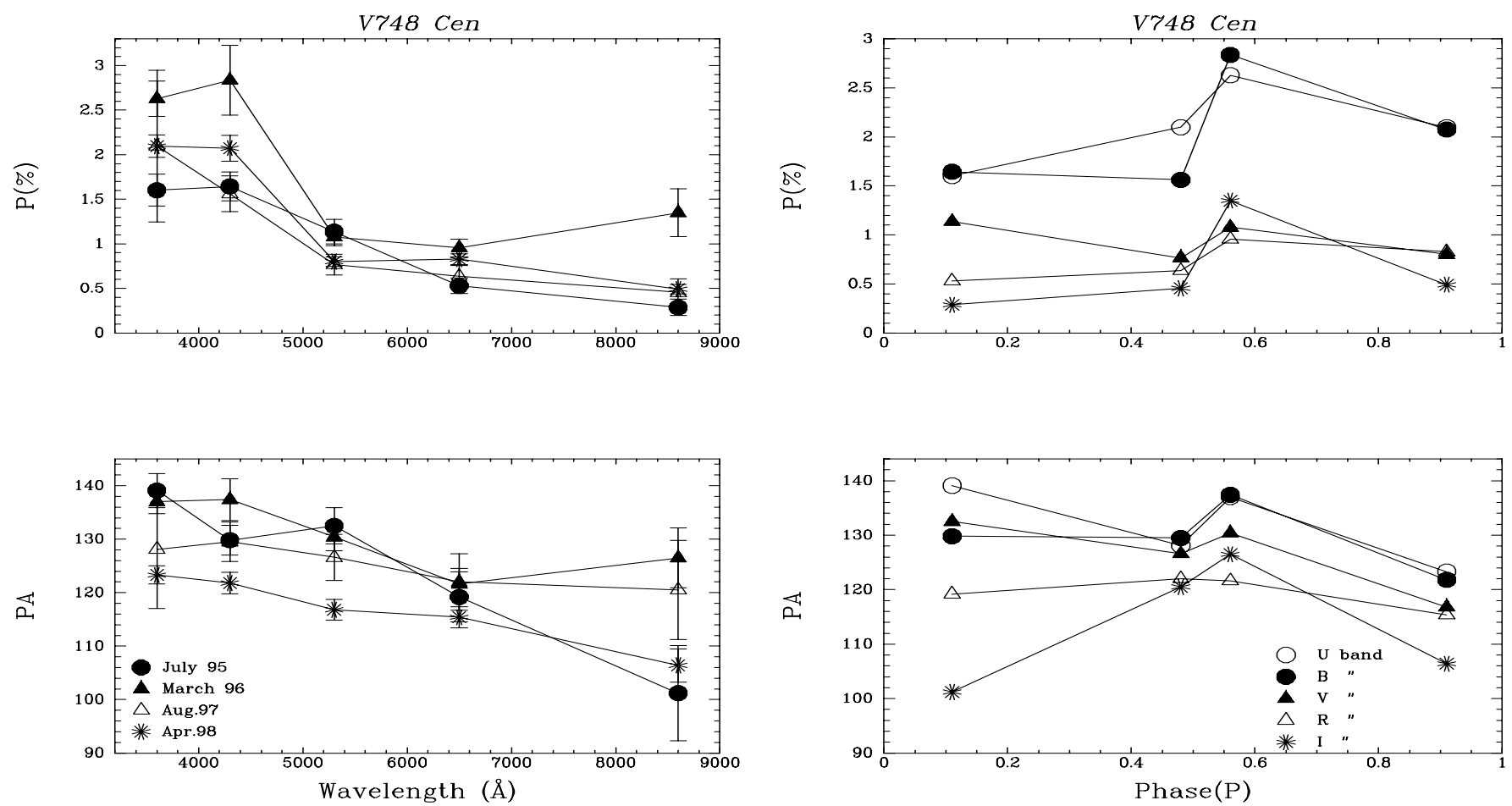

Fig. 1. V748 Cen: Wavelength dependence of linear polarization $P(\%)$ (top) and position angle (bottom) for different epochs

observed; this is typical for cool luminous stars, where polarization is produced by light scattering on dust particles in a circumstellar envelope. In July 1995 the amount of polarization increases to maximum values in the $U$ and $B$ bands. In March 1996, the wavelength dependence of the polarization remained the same as it was in 1995, but the amount of polarization increased from 1.6 to $2.6-2.8 \%$ in the $U B$ and from 0.3 to $1.3 \%$ in infrared. In August 1997 and April 1998, the polarization values in $U B$ are intermediate between those corresponding to the two first epochs while in $I$ they are similar to July 1995. In the $V$ and $R$ filters there were no significant changes with time.

The position angle shows rotations over the observed spectral range between $38^{\circ}$ (July 1995) and $8^{\circ}$ (August 1997). For each wavelength the rotation along the time is $16^{\circ}$ in $U B V$ bands and $7^{\circ}$ and $25^{\circ}$ in $R$ and $I$ bands, respectively.

The behavior of the polarization in V748 Cen can also be related with the orbital motion of the eclipsing binary, using the van Genderen et al. ephemeris,

$\operatorname{Min}(\mathrm{V})=$ JD $2441917+566.5 E$.

Figure 2 shows the polarization as a function of the phase. The percentage of polarization is greater in $U$ and $B$, increasing from $1.5 \%$ in phase 0.48 to $2.8 \%$ in phase 0.56 , when the hot component is moving towards the second quadrature suggesting that at least part of the polarization can be due to scattering of the light of the hot source due to the $\mathrm{M}$ giant envelope and the stream

Fig. 2. V748 Cen: Percentage polarization (top) and position angle (bottom) as a function of orbital phases for the $U B V R I$ filters

of material between the stars, such as it was proposed for CH Cyg by Piirola (1988a). If this is the case, around quadratures the line of sight is perpendicular to the line joining the stars and the scattering angles are favourable for producing a maximum of polarization, but unfortunately we have no observations at both quadratures. At phase 0.91 , before the primary eclipse when the cool giant is in front of the observer, the scattering angle is decreasing and a drop of polarization is observed.

\section{Hen 1103}

Hen 1103 was observed in July 1995, March 1996, August 1997 and April 1998 and our observations are the first polarimetric data of this object (Fig. 3). In July 1995 the degree of polarization reaches the minimum value in blue $(0.6 \%)$ and then it shows a relatively smooth increase to the larger wavelengths with a peak in $R$ of about $2.5 \%$. The wavelength dependence is reversed in March 1996, i.e., the polarization reaches the largest value of $3.7 \%$ and decreases slightly to the infrared.

The position angle is the same in all wavelengths, around $35-40^{\circ}$ and there is no rotation in the $B V R I$ range. However in July 1995 a rotation of $\sim 120^{\circ}$ between $U$ and $B$ is observed larger than the experimental error. This fact, i.e. the rotation over a small wavelength range, is a common phenomenon observed in other symbiotic stars cf. R Aqr (Schulte-Ladbeck 1985) and EG And 

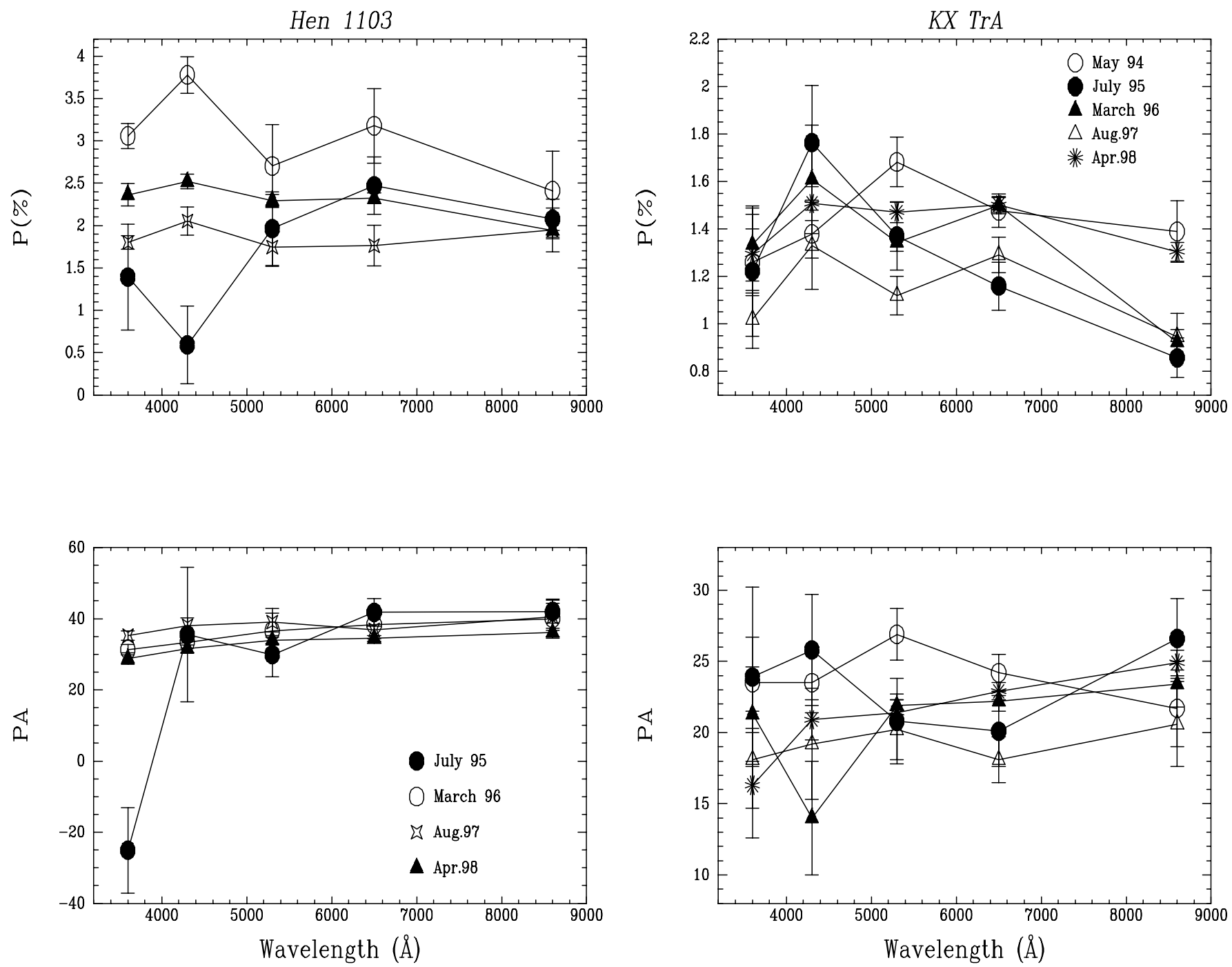

Fig. 3. Hen 1103: Wavelength dependence of linear polarization $P(\%)$ (top) and position angle (bottom) for different epochs

(SLAMS). It has been suggested that the observed rotation might be explained by scattering of the light of the two components, a red one and a blue one, by the same circumstellar region close to the two stars.

\section{KX $\operatorname{Tr} A=$ Hen 1242}

The rich emission-line spectrum of KX TrA resembles the one of the slow nova RR Tel (Webster 1966), in particular the evolution of the spectral excitation levels observed between 1965 and 1972 (Webster 1973). A wide range of ionization states for forbidden lines was observed, for example, $[\mathrm{Nev}]$ and [Fevir]. A light curve of KX TrA for the years 1889-1975 was given by Liller (1974), with quasiperiodic variations in $B$ between 10.1 and 13.6 magnitudes. This object seems to have a slow-rising outburst every 10-15 years and major outbursts during this interval were not recorded.

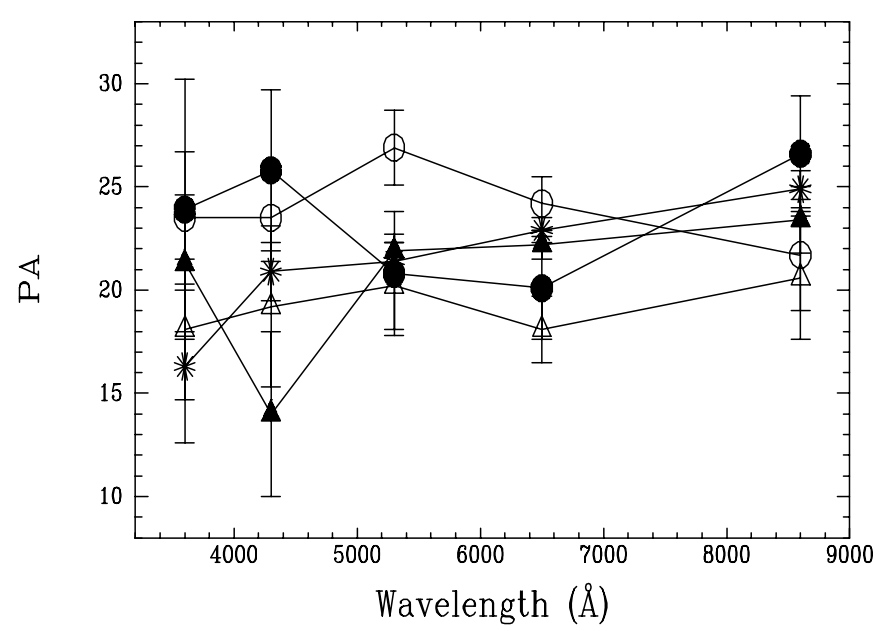

Fig. 4. KX TrA: Wavelength dependence of linear polarization $P(\%)$ (top) and position angle (bottom) for different epochs

SLAMS did not see evidence of an intrinsic polarization component in KX TrA from their observations carried out in July 1985 and April 1986. Moreover in two spectropolarimetric surveys (Schmid \& Schild 1994; Harries \& Howarth 1996) it was found that the amount and orientation of the continuum polarization outside the Raman lines $\lambda 6825$ and $\lambda 7082$ is small and practically constant in KX $\operatorname{TrA}$.

Our observations of KX $\operatorname{TrA}$ obtained during five observing runs, do not show any clear wavelength dependence of the polarization percentage neither of the position angle. The values are quite similar, within the error bars (Fig. 4), although it is worth noticing that the degree of polarization in $I$ during May 1994 and April 1998 is $~ 1.4 \%$ while in July 1995, March 1996 and August 1997 decreases to $\sim 0.9 \%$. 

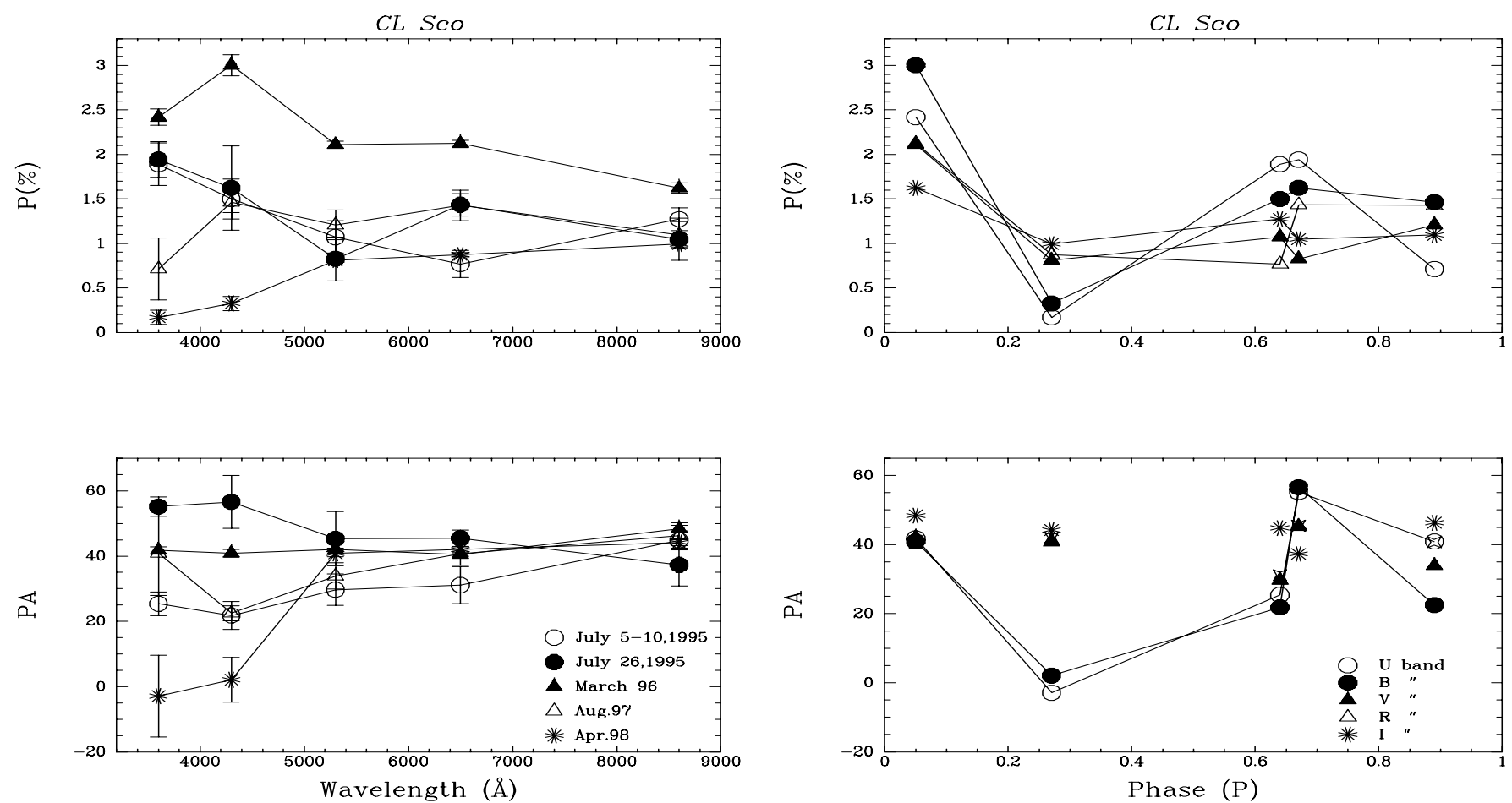

Fig. 5. CL Sco: Wavelength dependence of linear polarization $P(\%)$ (top) and position angle (bottom) for different epochs

\section{Scorpii}

Kenyon \& Webbink (1984) suggested that the low excitation symbiotic star CL Sco is a system similar to CI Cyg, containing a disk-accreting main sequence star. They found that the well observed optical minima of this system can be represented by the ephemeris:

$\operatorname{Min}(\mathrm{pg})=2427020+624.7 E$.

For CL Sco we have not found any published polarimetric data. According to our observations shown in Fig. 5, at the time of the first observing run between July 611, 1995, CL Sco presented a decreasing trend in the degree of polarization with a peak of approximately $1.9 \%$ in the $U$ band and the position angle showed an overall rotation between ultraviolet and infrared amounting to about $15^{\circ}$. Twenty days later, on July 27 , the polarization increased in the red from $0.8 \%$ to $1.4 \%$ but changes in opposite sense were observed in the orientation with a rotation of $\sim 20^{\circ}$.

In March 1996, the polarization degree increased in all spectral regions, particularly in $B$ passband where it doubled with respect to July 1995 whereas the position angle remained constant at $\sim 45^{\circ}$ in all wavelengths. In August 1997, the wavelength dependence is similar to the one of March 1996 but the values are smaller, specially in $U$ and $B$ where the polarization has decreased between $0.5-0.7 \%$. Finally, in April 1998, the polarization shows a greater variation, increasing from $P(U) \sim 0.2 \%$ to $P(R) \sim$ $0.9 \%$ whereas the position angle also shows a strong

Fig. 6. CL Sco: Percentage polarization (top) and position angle (bottom) as a function of orbital phases for the $U B V R I$ bands

rotation between $U B$ and $V$ of about $40^{\circ}$. In general we can see that the ultraviolet and the blue light show the larger variations in the percentage of polarization and stronger rotations in the orientation.

The behavior of polarization vs. the orbital motion is shown in Fig. 6 and it seems to be very different from that of V748 Cen. The larger polarization is found at phase 0.05 , when the components are in conjunction with the giant in front while near the first quadrature at phase 0.27 , when the line of sight is perpendicular to the binary axis, a minimum in the polarization together with a strong rotation are observed. Between the phases 0.66 and 0.9 the ultraviolet polarization decreases but important information during the second quadrature is lacking.

\section{AR Pavonis}

It is the first eclipsing binary discovered among the symbiotic stars (Mayall 1937). The characteristics of this system remain still elusive and several models have been proposed. According to Thackeray \& Hutchings (1974) the system is constituted by a cool giant (M3-4 III-II) filling its Roche lobe and losing mass towards an evolved hot component. The eclipses seem to be due to the occultation of a dense part of a nebula surrounding the hot star rather than to a stellar body. Kenyon \& Webbink (1984) modified this model suggesting that the hot component is a main sequence star with an accretion disk. The shape 

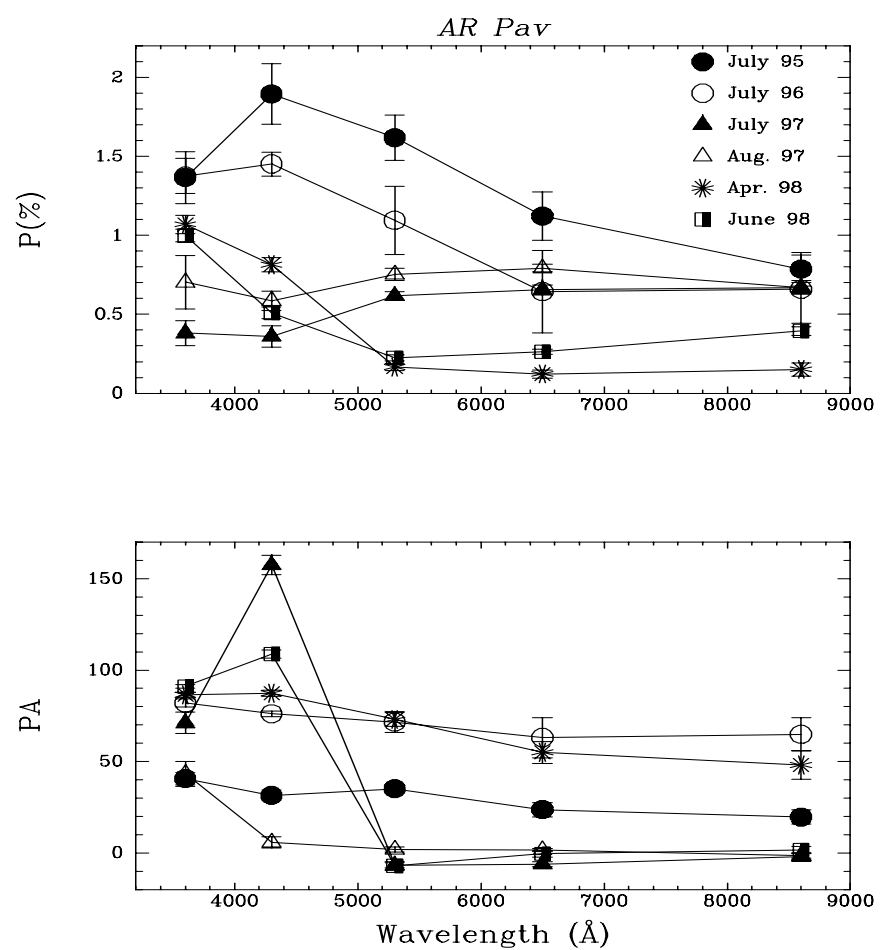

Fig. 7. AR Pav: Wavelength dependence of linear polarization $P(\%)$ (top) and position angle (bottom) for different epochs

of the eclipses in the light curve was analyzed by Bruch et al. (1994) and AR Pav seems to be highly variable out of eclipse due to a modulation of the mass transfer from the red giant to the hot component. Skopal et al. (1997), re-analyzed the historical light curve (1888-1996) of AR Pav. During the quiescent stages the observations can be accounted for by a large accretion disk around the hot component but they proposed that at the outbursts an opposite mass flow from the hot component towards the cool giant occurs giving rise to a collisional emission region on the giant surface.

SLAMS observed polarimetrically AR Pav on two occasions and considered this star as a borderline case. Our polarimetric observations were carried out during six epochs, July 1995, July 1996, July 1997, August 1997, April 1998 and June 1998. Important changes in the wavelength dependence are observed both in the percentage of polarization and the position angle, such as it is shown in Fig. 7. We can distinguish three kinds of the wavelength dependence. The first occurs in July 1995 and July 1996; it shows a polarization with a maximum in $B$ and decreasing to red. On the contrary, the second one shows a minimum in $B$ (July 97-August 97) and finally, the third one (April 98-Jun. 98) displays a high polarization in ultraviolet decreasing to $V$ while the $V R I$ bands show the smallest values of polarization. The position angle curves indicate slight rotations with the wavelength in each one of the four observation sets but in July 97 and April 98 strong rotations in $U$ and $B$ of more than $90^{\circ}$ are evident. The strongest temporal variations in the position angle
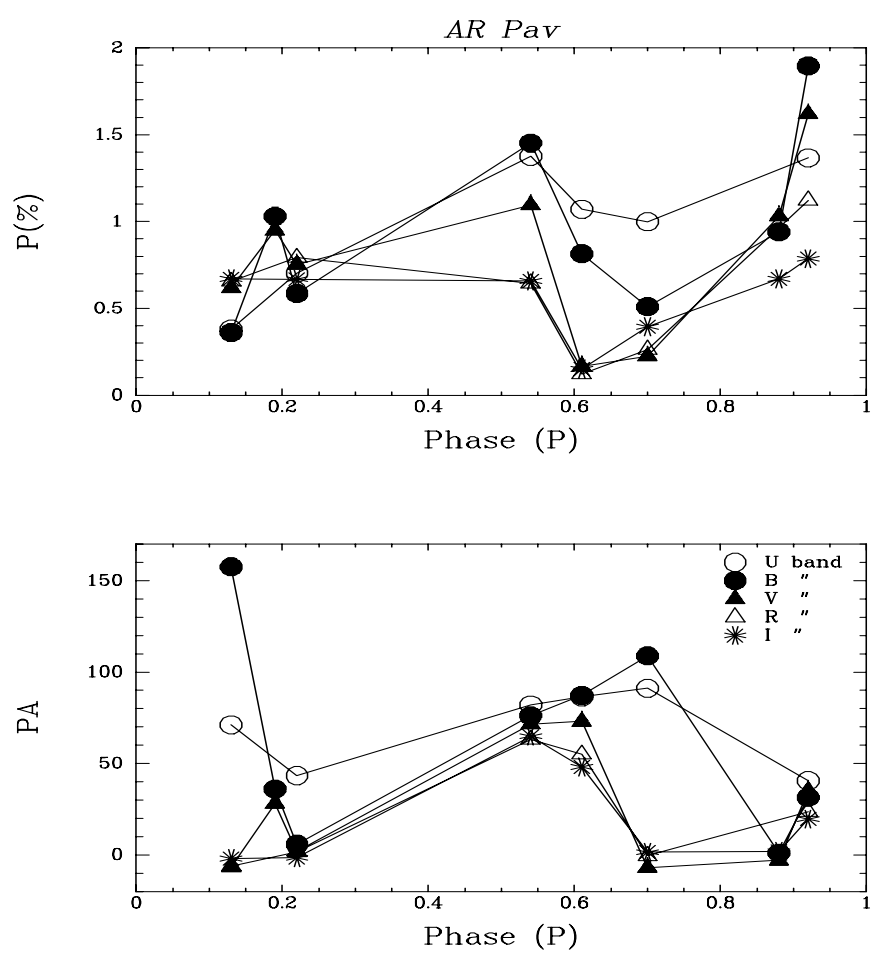

Fig. 8. AR Pav: Percentage polarization (top) and position angle (bottom) as a function of orbital phases for the $U B V R I$ filters. The published values given by SLAMS are included at phases 0.19 and 0.88

correspond also to the $B$ band with a time scale as short as 54 days (July 97-August 97).

Adopting the ephemeris given by Bruch et al. (1994):

Min $=$ JD2 $420331.3+604.5 E$,

we analyse the polarization of AR Pav vs. phase in order to know if the complicate polarimetric behavior of this system is correlated with the orbital motion. First of all, the three quoted wavelength dependences concerning the percentage of polarization correspond to three different positions of the component stars with respect to the observer, namely the two conjunctions, before the first quadrature and before the second quadrature, respectively. We can see in Fig. 8 that the polarization reaches the highest values in $U B(1.4-1.9 \%)$ in phase 0.92 (July 95) close to the eclipse, when the cool component is in front of the observer. At phase 0.53 (July 96), immediately after the second conjunction when the hot component is in front, another increase of polarization $\sim 1.5 \%$ in $U B$ is observed. Simultaneously, the orientation of the position angle at both conjunctions shows a rotation of $\sim 40^{\circ}$. Moreover, at phase 0.60 (April 98) the polarization in $V R I$ presents the minimum observed values, $\sim 0.2 \%$ when the hot star is going away from the observer. At phases 0.19 and 0.88 (SLAMS's data) the position angles in $U$ are offset by $\sim 40^{\circ}$ and $\sim 90^{\circ}$ from the $V R I$ bands respectively.

Khudyakova (1988) found that variations of polarization in CI Cyg and R Aqr reflect the orbital motion 

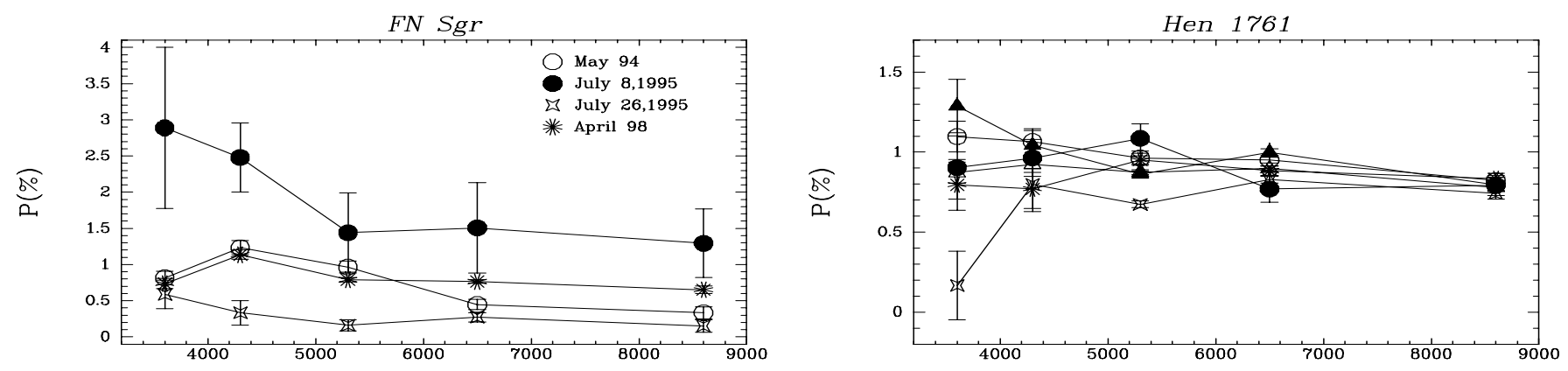

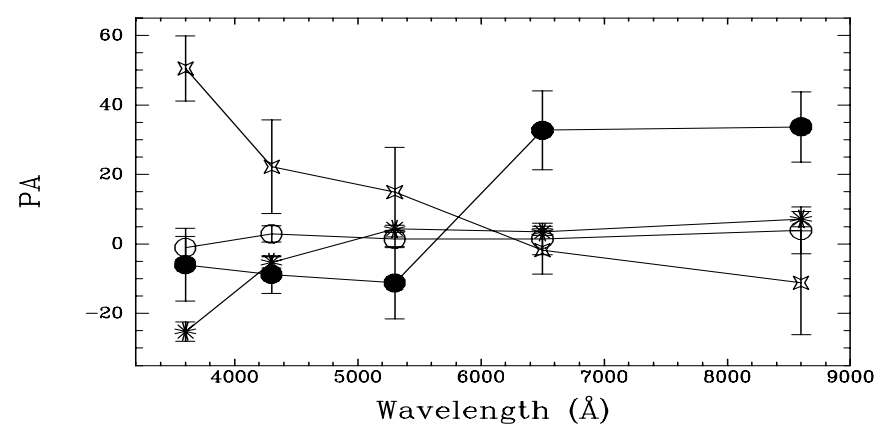

Fig. 9. FN Sgr: Wavelength dependence of linear polarization $P(\%)$ (top) and position angle (bottom) for different epochs

and the maxima lie near the conjunction when the hot component is between the cool star and the observer.

\section{FN Sagitarii}

This typical S-type symbiotic star has undergone two outbursts ( $\delta m \sim 4 \mathrm{mag}$ ) in 1924-26 and 1936-41. Semiregular variations with smaller continuous fluctuations are superimposed (Kenyon 1986 and references therein). Highexcitation emission lines of HeII, [OIII] and [NeIII] are present in FN Sgr; in addition, [FevI], [FevII] and the Raman scattering band at $\lambda 6825$ have been reported by Barbá et al. (1992).

Our data were obtained in May 1994, July 9, 1995, July 27, 1995 and April 1998 and there are no previous polarimetric observations of FN Sgr. Conspicuous changes in the amount of polarization for all the wavelengths were observed, specially during the two sets of observations of July 1995 (Fig. 9). The maximum values correspond to $2.9 \%-2.5 \%$ in $U B$ bands on July 8 but after eighteen days the polarization suddenly decreases in all bands reaching $0.6 \%-0.3 \%$ whereas rotations in opposite sense between $U B V$ and $R I$ bands were detected. These changes in the position angles were $\sim 140^{\circ}$ on July 9 and $\sim 40^{\circ}$ on July 27. In May 94 and April 98 the polarization presents a maximun in $B(\sim 1.2 \%)$ and then decreases slightly to the infrared. The position angles are very similar during those epochs and a small rotation is detected along the wavelengths.

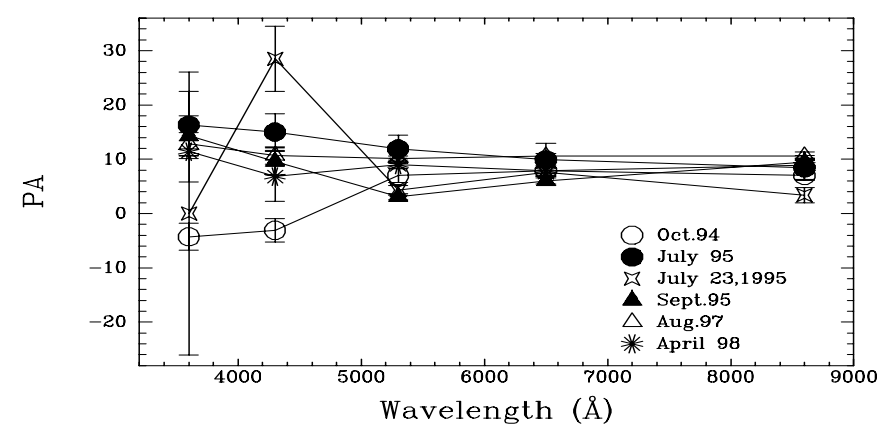

Fig. 10. Hen 1761: Wavelength dependence of linear polarization $P(\%)$ (top) and position angle (bottom) for different epochs

Visual magnitudes and $V$ photometry collected in the period 1977-1998 (Belczyński \& Mikolajewska, private communication) shows that the FN Sgr light curve presents systematic brightness decline with moderate periodic-like light changes, and one major amplitude started in 1995. In July 1995 we obtained the most remarkable variations in the polarimetric parameters in a short time scale of eighteen days. All our observations correspond to the large amplitude in the active state of the light curve.

\section{Hen 1761}

Hen 1761 is an infrequently studied symbiotic star. During the period 1990-1992 eruptive characteristics were detected in its spectra and the quiescence phase started in 1993 (Brandi et al. 1998).

SLAMS measured the polarization of Hen 1761 in the bands $B V I$ and $\mathrm{H} \alpha$. The results indicated that probably there is polarization, intrinsic to the object.

We carried out the observations in six different runs (see Table 2). The dependence of the polarization degree with the wavelength is not very conspicuous and the variations are inside the error bars. The most important variations take place on July 24, 1995 when the $U$-filter polarization decreases from $\sim 1.3 \%$ to $0.2 \%$ and the position angle rotates less than $30^{\circ}$ (Fig. 10) in $U B$. 


\section{RR Telescopii}

RR Tel underwent a nova-like outburst that started in 1944. It was classified as D-type symbiotic system; infrared observations show the presence of a Mira variable as cool component with long-period pulsations. As RR Tel has declined from light maximum, the optical spectrum has slowly developed high ionization lines. A rich emission line spectrum is superimposed on a weak continuum.

Many authors have observed RR Tel polarimetrically but contradictory results have been obtained. SchulteLadbeck \& Magalhães (1987) found that RR Tel did not show intrinsic polarization. Spectropolarimetric observations of the emission lines at $\lambda 6825$ and $\lambda 7082$ as due to Raman-scattered Ovi $\lambda \lambda 1032$ and 1038 resonance lines were carried out by Schmid \& Schild (1994); Espey et al. (1995) and Harries \& Howarth (1996). The measurements of Schmid \& Schild in the range $6700-7500 \AA$ together with the polarization map of surrounding stars, allowed to infer that an intrinsic component of polarization was present in RR Tel. Moreover, the mean continuum polarization and position angle over the range $6400-7200 \AA$ Abtained by Espey et al., indicated that the continuum polarization is predominantly due to interstellar dust grains. The continuum polarization data given by Harries \& Howarth showed a significant difference with the red region photopolarimetric measures given by SchulteLadbeck \& Magalhães (1987) and Schmid \& Schild (1994). This temporal variability was an evidence of intrinsic polarization in RR Tel.

As it is seen in Fig. 11, our multifrequency observations in October 1994 and July 1995 show the percentage of polarization with a slow decrease with increasing wavelengths. However, in August 97 the polarization presents an approximately flat wavelength dependence around $0.3-0.4 \%$ and it rises with the wavelength in April 98 from $0.5 \%$ in $U$ to $0.8 \%$ in $I$.

The position angle in $U B V R$ shows none or small temporal variations; the rotations in these filters are less than $\sim 20^{\circ}$. However a conpiscuous behavior is seen in the $I$ band where a change in the orientation of about $60^{\circ}$ between the first two and last two observation sets is evident. There is also a small rotation of $\sim 15^{\circ}$ between October 94 and July 95. Consequently, we can confirm the existence of intrinsic polarization in RR Tel.

$\mathrm{RR}$ Tel is the only D-type symbiotic included in our sample with a Mira variable as cool component. An interesting point is to make a more detailed study of the temporal variation of polarization in this object, in order to find a correlation with the Mira phase such as it was found for R Aqr (Aspin \& Schwarz 1988 and references therein); UV Aurigae (Khudyakova 1985, 1988) and o Ceti (Shawl 1975).

As it is quite normal for Mira variables, the light curve of $\mathrm{RR}$ Tel is not strictly repetitive over long timescales. Therefore, for the determination of phases, we
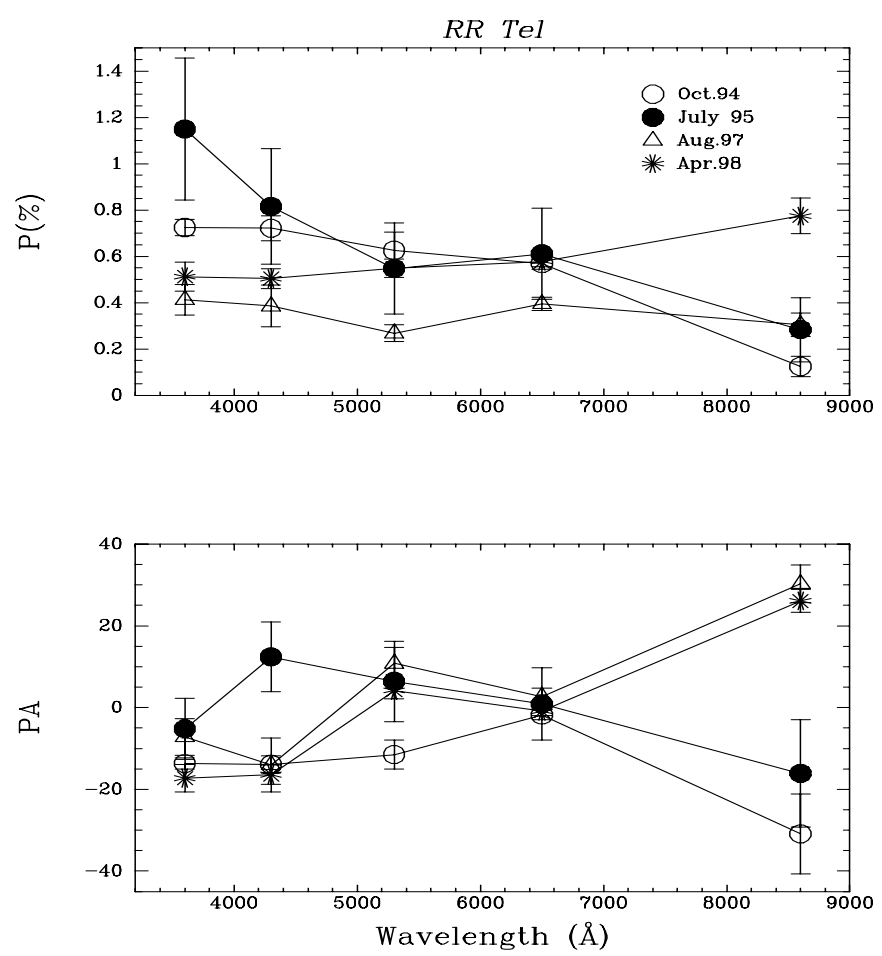

Fig. 11. RR Tel: Wavelength dependence of linear polarization $P(\%)$ (top) and position angle (bottom) for different epochs

used an epoch of maximum light obtained from JHKL photometric data collected by Feast et al. (1983) during 1975-81 and the period of 387 days. Figure 12 shows the variation of the degree and angle of polarization as a function of the phases. We have included the polarimetric observations of RR Tel given by Schulte-Ladbeck \& Magalhães (1987) in the UBVI-bands at phase 0.46; by Schmid \& Schild (1994) and Espey et al. (1995) in the $R$-band at phases 0.03 and 0.57 respectively and by Harries \& Howarth (1996) in the $I$-band at phase 0.79 . Inspection of the $U B V R$ polarization variations are not consistent with the phase of brightness variation of the Mira, but the $I$-band however, presents the percentage of polarization with an increasing trend from the maximum light (phase 0.0) reaching the maximum of polarization $(0.8 \%)$ around phase 0.5 . This behavior with the light variations is in the same sense of R Aqr (Schwarz \& Aspin 1988) but opposite to those of UV Aur (Khudyakova 1985) and o Ceti (Shawl 1974). The variable infra-red polarization modulated with the Mira phase indicates that at least one polarigenic mechanism in operation in $\mathrm{RR}$ Tel is intrinsic to the cool component and like the majority of late-type variable stars, the polarization can be explained by scattering by grains in asymmetric circumstellar nebula around the Mira. On the other hand, position angle for the $I$-band shows a total rotation of $\sim 70-90^{\circ}$ with cyclic fluctuations with the phases which would correspond to changes in the effective scattering angle along the orbital motion. If this argument is correct 

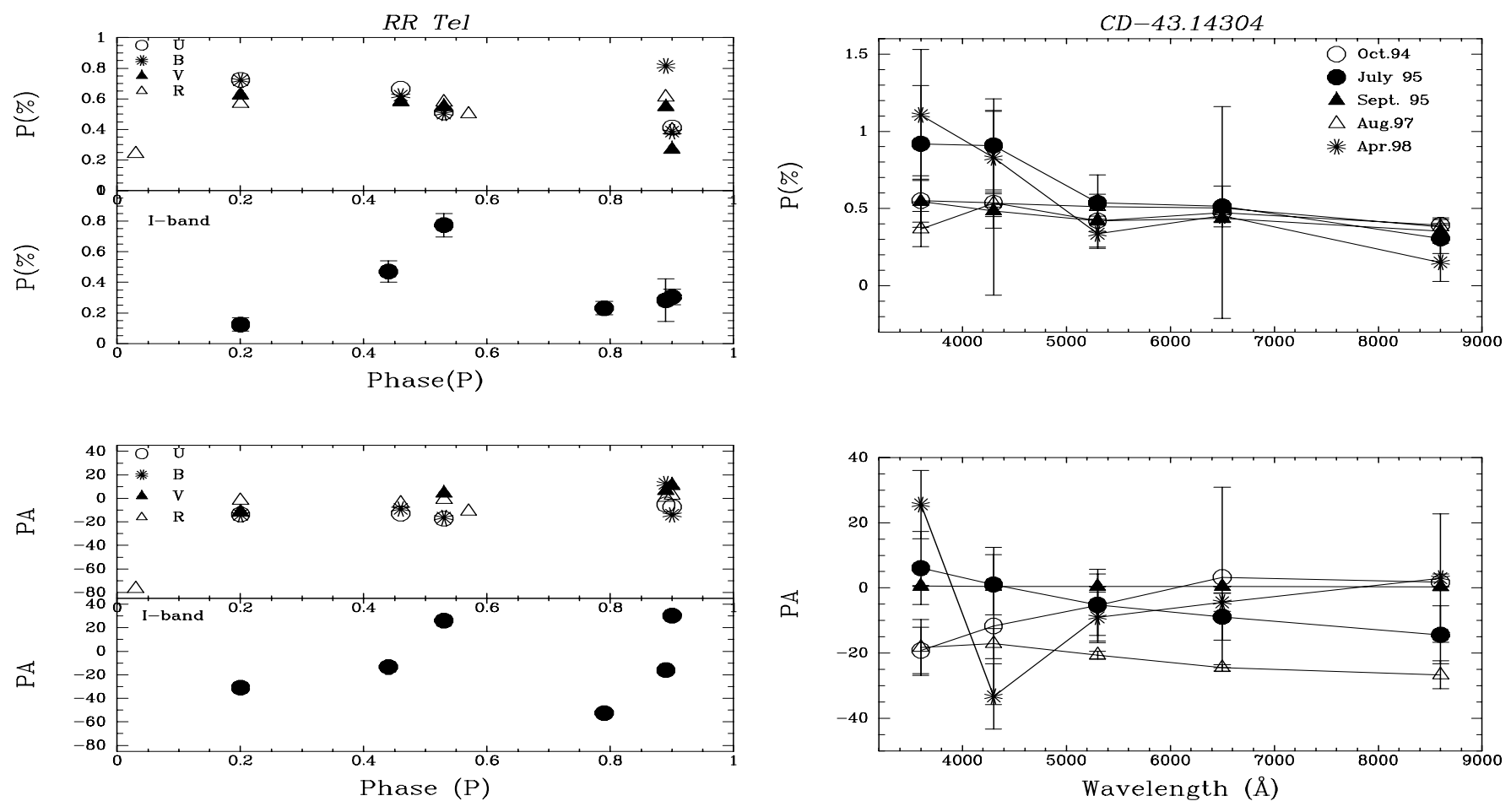

Fig. 12. RR Tel: The polarimetric variations of $R R$ Tel with the Mira phase. From top to botton the figures show the $U B V R$ band polarization variations, The $I$-band polarization variations, the $U B V R$ band position angle and the $I$-band position angle. We have included the polarimetric observations of RR Tel given by Schulte-Ladbeck \& Magalhães (1987) in the $U B V I$-bands at phase 0.46; by Schmid \& Schild (1994) and Espey et al. (1995) in the $R$-band at phases 0.03 and 0.57 respectively and by Harries \& Howarth (1996) in the $I$-band at phase 0.79

and taking into account the distribution of the position angles with the Julian days, one can approximate an orbital period of $\simeq 10 \mathrm{yr}$ for RR Tel.

\section{CD $-43^{\circ} 14304$}

The published polarimetric observations of CD $-43^{\circ} 14304$ are spectropolarimetric studies of the Raman scattered emission lines at $\lambda \lambda 6825$ and 7082 carried out by Schmid \& Schild (1994) and Harries \& Howarth (1996). The continuum polarization values in the red region presented by those authors are in agreement within the errors; no temporal variations were detected. Moreover, by means of the polarization map of surrounding field stars, they concluded that the continuum polarization at $\sim 7000 \AA$ for CD $-43^{\circ} 14304$ is probably dominated by the interstellar polarization. Our observations confirm the temporal invariance observed in the polarization in the $V, R$ and $I$ bands (see Fig. 13) but it is not the same for the ultraviolet and blue; there is a maximum of about $1 \%$ in July 95 and April 98 and the polarization falls to $0.5 \%$ or less during the other sets of observations. The wavelength

Fig. 13. CD $-43^{\circ} 14304$ : Wavelength dependence of linear polarization $P(\%)$ (top) and position angle (bottom) for different epochs

dependence of the position angle seems to be variable along the time. Moreover, there are small rotations in the position angle among the different filters. A change between $20^{\circ}-30^{\circ}$ occurs in all the wavelengths for different epochs and strong rotations of $\sim 60^{\circ}$ in $U B$ and $\sim 30^{\circ}$ in $B V$ are observed in April 98.

Schmid et al. (1998) have obtained the radial velocity curve for the cool component in CD $-43^{\circ} 14304$ and an orbital period of 1448 days for circular orbit of the binary was determined. Using their ephemeris corresponding to the moment of maximum velocity,

$\operatorname{JD}(\operatorname{Max})=2445929+1448 E$,

the orbital phases of $\mathrm{CD}-43^{\circ} 14304$ observations were calculated and correlated with the polarization degree and position angle such as it is shown in Fig. 14. The larger polarization in $U(1.1 \%)$ is seen at phase 0.45 before the quadrature when the giant is approaching to the observer and the line joining the stars is perpendicular to the line of sight. Simultaneously, remarkable rotations in $U$ and $B$ bands are observed. When the giant is in front of the observer (phase 0.75 ) the polarization in $U B$ increases again to $0.91-0.92 \%$ but smaller rotations occur.

\section{AG Pegasi}

AG Peg is a well studied symbiotic binary which contains a normal M2 giant and a compact object. A nova-like 

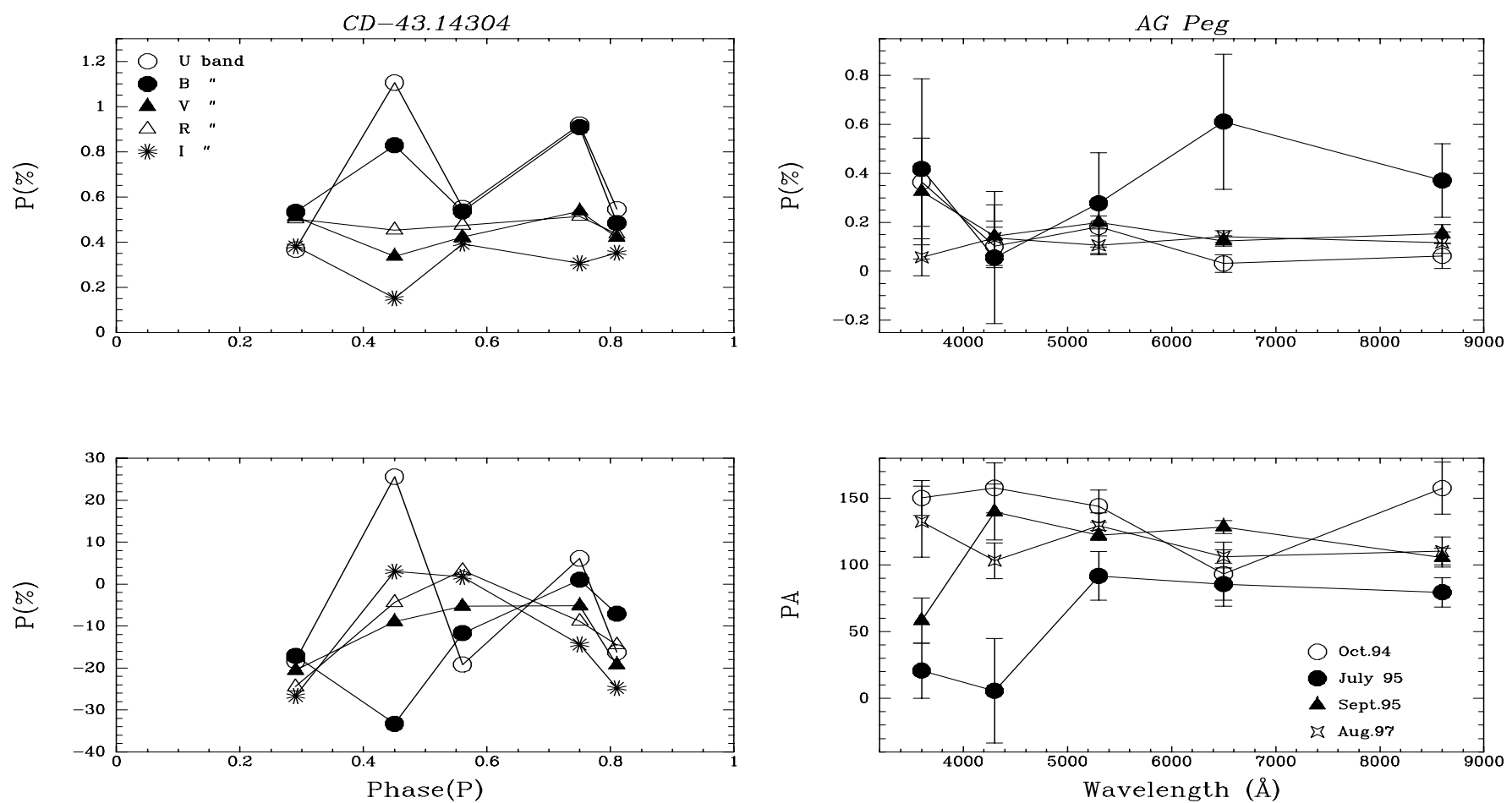

Fig. 14. CD $-43^{\circ} 14304$ : Percentage polarization (top) and position angle (bottom) as a function of orbital phases for the $U B V R I$ filters

outburst began in 1850 and a very slow decline has taken place since 1871. At the moment AG Peg may be close to the end of its outburst. The spectroscopic orbital period of the binary (800 days) was discovered by Merrill (1929a, 1929b) and spectroscopic orbits were also determined by Cowley \& Stencel (1973); Hutchings et al. (1975); Slovak \& Lambert (1988) and Garcia \& Kenyon (1988). Belyakina (1970) showed that irregular 0.3 mag fluctuations in $V$ followed the spectroscopic period and that those variations of brightness could be caused by the reflection effect. According to this model, ultraviolet radiation from the hot component heats up the facing hemisphere of the giant, causing the cool star to radiate more energy when its heated hemisphere faces the observer. The photometric period was improved by Meinunger (1981); Belyakina (1985); Fernie (1985) and Luthardt (1990).

AG Peg was studied polarimetrically by other authors, Serkowski (1970); Piirola (1983); Schulte-Ladbeck (1985) and SLAMS. Variations in the intrinsic polarization were detected as far as the wavelength dependence and the time are concerned. In all the cases the polarization values were rather small, peaking $\sim 1 \%$ in $B$ (Serkowski 1970) and large rotations of the position angle with wavelength were also observed, the most remarkable being $90^{\circ}$ between $3699 \AA$ and $6500 \AA$ and $170^{\circ}$ at $8000 \AA$ (Aspin \& Schwarz 1988).

Our observations of October 1994 show polarization decreasing slightly with wavelength from a maximum of

Fig. 15. AG Peg: Wavelength dependence of linear polarization $P(\%)$ (top) and position angle (bottom) for different epochs

$0.4 \%$ in $U$ to a minimum of $0.06 \%$ in $R$ and $I$ (Fig. 15). Similar behavior is observed in September 95 but in July 1995, although the data have relatively large uncertainties in this epoch; the wavelength dependence changes, increasing the polarization to $0.6 \%$ in $R$ and $0.4 \%$ in $I$. In August 97 the polarization decreases until $0.06 \%$ in $U$ but in the other filters the values are similar to those of October and September. In the different epochs a constant value of $\sim 0.1 \%$ in $B$ band is observed. The position angle shows remarkable rotations. In October 94 a dip in $R$ indicates a rotation of $\sim 60^{\circ}$ between $U B V$ and $R$ and between $R$ and $I$. In July 95 the rotation of $\sim 90^{\circ}$ between $U B$ and $V R I$ is associated with an abrupt decreasing to $0.06 \%$ in the polarization. In September 95 is $\sim 80^{\circ}$ between $U$ and $B$ whereas in the last data set of August 97 a small rotation of approximately $20^{\circ}$ from $U$ to $I$ is observed. Such a large rotation in the position angle along the wavelengths has been already detected in AG Peg by other authors, as it was indicated above.

In particular and according to Daniel (1982), a change in the orientation by $90^{\circ}$ in the wavelength dependence was found indicative of the polarization reversal in bipolar nebula. AG Peg has an optical nebula (Fuensalida et al. 1988) and an extended and complex nebula detected at radio (Hjellming 1986; Kenny et al. 1991). The structure surrounding AG Peg detected in $\mathrm{H} \alpha$ shows two lobes resolved at $5^{\prime \prime}$ and $4^{\prime \prime}$ from the central part in virtually opposite directions approximately NW-SE. 

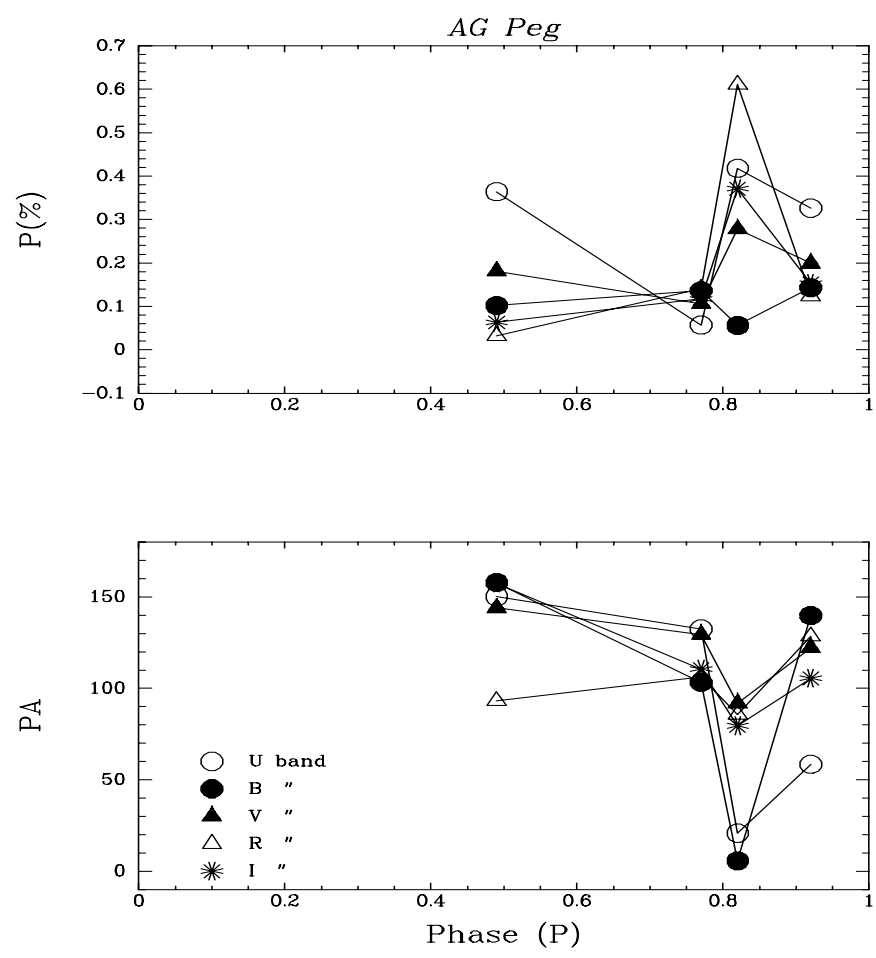

Fig. 16. AG Peg: Percentage polarization (top) and position angle (bottom) as a function of orbital phases for the $U B V R I$ filters

The Fig. 16 shows our results of polarization as a function of orbital phase. We calculate the phases adopting Fernie's (1985) ephemeris,

$\operatorname{Min}(\mathrm{B})=\mathrm{JD} 2442710.1+816.5 \mathrm{E}$.

Unfortunately our observations are concentrated in the second half of the orbital cycle.

Taking into account the proposed model (Belyakina 1970), in October 94, phase 0.49 corresponds to the moment when the cool star is in front of the observer close to the conjunction with the other star. Here the ultraviolet polarization is more important and then decreases until phase 0.77 near the quadrature, where in all the wavelengths, the polarization reaches similar values (about $0.1 \%)$. An abrupt increase in the polarization, specially at $U, R$ and $I$, is observed at phase 0.82 when the giant is going away from the observer. This fact is accompanied by bigger changes in the position angle. Finally, on September 95 , at phase 0.92 the system is near the first conjunction, when the hot hemisphere of the giant is in front of the observer and the polarization decreases in $U V R I$ bands. We can mention that although the blue polarization is nearly constant along the phases, it shows the strongest rotation of the position angle at phase 0.82 .

It is very difficult to bring out conclusion about the behavior of polarization in AG Peg, as far as the wavelength dependence and the orbital motion are concerned. It seems that several polarigenic mechanisms are working producing different polarization in the ultraviolet, blue and longer wavelengths. Perhaps a superposition of mechanisms would give rise to such complicated results.

\section{Discussion}

In eight out of ten symbiotic stars of the sample, our polarimetric results show different wavelength dependences of the polarization percentage and/or the position angle and temporal variations. Therefore, we can conclude that intrinsic polarization is a common characteristic of this group of stars. The presence of intrinsic polarization in KX TrA and Hen 1761 is not conclusive. We find wavelength dependences of $P(\%)$ decreasing monotonically from blue to infrared; decreasing in opposite sense; peaking in some particular wavelength or presenting a dip in the visual and then increasing to the blue and to the red. Therefore, there is not any particular wavelength dependence of the polarization on the contrary, different trends are observed for each star along the time. Moreover, no conclusive correlation between the behavior of polarization and position angle can be drawn. In the observed objects it is common to find that the same wavelength dependence in the degree of polarization is associated with different orientations in the position angle.

In several systems we can see that the most important changes in the percentage of polarization along the time are observed towards the short wavelengths, whereas the smaller variations or constant values are observed in the $I$ band, which is the cases of Hen 1103, CL Sco, AR Pav and CD $-43^{\circ} 14304$. As it can be seen, Hen 1103 and CL Sco present the particularity that both the polarization degree and the position angle are practically constant in the infrared. The blueward slope of the $P(\%)$ trend is highly variable even if considering the same object (see V748 Cen, CL Sco, AR Pav, RR Tel and CD -43 $\left.{ }^{\circ} 14304\right)$.

It is known that the dust richest D-type symbiotics are intrinsically polarized (Schmid 1997). Since dust scattering is considered the main source for intrinsic linear polarization in symbiotic systems, our positive results obtained for seven S-type objects in the sample, would suggest the presence of dust surrounding asymmetrically the system or the cool component of S-type symbiotics which does not show infrared excess. This would be in agreement with results obtained from polarimetric studies of red variable stars: the polarizing particles can be placed near the star while the particles producing the infrared excess are farther from the star (Shawl 1975) or different grains may be involved in producing the polarization and the infrared excess (Coyne \& Magalhães 1977).

In this work RR Tel is the only D-type symbiotic star with a Mira variable as cool component. We confirm the intrinsic polarization of this object, especially in the $I$-band which seems to be associated to the Mira period, corresponding to increasing polarization with decreasing brightness. More polarimetric observations of this object 
would be particularly necessary to isolate the mechanisms occurring at shorter wavelengths and to confirm the modulation of the infra-red polarization with the Mira period and, to some extent, the binary period.

Some symbiotic systems display extended ionized nebulae with different morphological structures (bipolar, elliptical, irregular, jet-like). The polarimetric study of these objects is particularly interesting because it allows one to correlate the orientation of polarization vector with the known nebular geometry and consequently the scattering region can be defined (for example in BI Cru, Harries 1996). Corradi et al. (1999) have presented an optical search for extended nebulae around symbiotic stars and a compilation of objects with nebulae detected at optical and radio wavelengths, as well as a list of optical negative detection. AG Peg is the only star of this work included in their lists of radio and optical detections. As we mentioned, some features of polarization in this object seem to be related with the bipolar nebulae, especially the large rotations of the position angle. However, scattering regions connected to the binary system might be present and more observations along the period would be necessary in order to draw firm conclusions. Hen 1103, KX TrA and RR Tel have no nebula detections according to Corradi et al. (1999); it means that the observed polarization is originated in gas structures close to the stars or inside the binary (stellar photosphere, gaseous stream, accretion disks). There is no information about the existence of extended nebulae in the remaining S-type symbiotics included in our sample, although Corradi et al. (1999) have confirmed less frequent ocurrence of extended nebulae among S-type objects.

As regards to the correlation between the polarization and the orbital phase analysed in the other symbiotic binaries with known periods (V 748 Cen, CL Sco, AR Pav and CD $\left.-43^{\circ} 14304\right)$, the scarse number of observations allows one only to glance at the behavior of the polarization in particular at positions of both components, respect to the line of sight. V 748 Cen shows an increase of polarization when the hot component is moving towards the second quadrature and a decrease before the primary eclipse when the giant is in front of the observer. In this case, like for $\mathrm{CH} \mathrm{Cyg}$, the polarization can be due to scattering of the light of the hot source by the $\mathrm{M}$ giant envelope and/or from the stream of material between the stars. $\mathrm{CD}-43^{\circ} 14304$ shows also an increasing $U B$ polarization before the first quadrature but we have no observations during the second one. On the other hand, CL Sco and AR Pav show an opposite behavior of polarization with phases. The higher values of $P(\%)$ occur when the components are in conjunction whereas we have a minimum in the polarization when the line of sight is perpendicular to the binary axis. Strong rotations in the position angle are observed in both cases.

\section{Conclusions}

The following general conclusions can be drawn:

i) We present new polarization measurements of ten symbiotic stars and analyze the wavelength dependence of both the percentage of polarization and the position angle. We find that eight objects show temporal variations vs wavelength, indicating that intrinsic polarization is present (V748 Cen, Hen 1103, CL Sco, AR Pav, FN Sgr, RR Tel, CD $-43^{\circ} 14304$ and AG Peg). Four out of these polarized stars did not possess polarization data before the present study (V748 Cen, Hen 1103, CL Sco and FN Sgr). KX TrA and Hen 1761 represent two cases of unpolarized symbiotics.

ii) Except for RR Tel, 7 of the polarized objects are S-type symbiotics. If the polarization is produced mainly by dust particles, the circumstellar envelope surrounding the cool component in S-type symbiotics contains grains different from those producing infrared excess.

iii) We present wavelength dependences of $P(\%)$ decreasing monotonically from blue to infrared; decreasing in opposite sense; peaking in some particular wavelength or presenting a dip in the visual and then increasing to blue and red. Different dependences can be observed for each star along the time. Moreover, no conclusive correlation between the behavior of polarization and position angle can be drawn.

iv) The time scales of variations are of the order of several days or months.

v) In five cases (V748 Cen, CL Sco, AR Pav, CD $-43^{\circ} 14304$ and AG Peg) the orbital period of the binary is known and a correspondence between the orbital phase and the variable behavior of the polarization might be suggested in some of them. However, the maxima and minima in the polarization are produced at different configurations of the components with respect to the observer. Consequently, the source of light and the scattering regions are not the same in all the binary systems. It is necessary to extend the observations in time in order to cover the orbital cycles.

vi) Several scattering regions associated with gas stream, accretion disks, Mira component or extended nebulae could be present and different sources of intrinsic polarization should be considered in the same object.

\section{References}

Aspin C., Schwarz H.E., 1988, Polarized Radiation of Circumstellar Origin, Coyne G.V. et al. (eds.). Univ. of Arizona Press, p. 403

Barbá R., Brandi E., García L., Ferrer O., 1992, PASP 104, 330

Belyakina T.S., 1970, Astrofizika 6, 49

Belyakina T.S., 1985, IBVS No. 2697

Brandi E., Barbá R., García L.G., Beltrán N., 1998, A\&AS 132,281 
Bruch A., Niehues M., Jones A.F., 1994, A\&A 287, 829

Corradi R.L.M., Brandi E., Ferrer O.E., Schwarz H.E., 1999, $A \& A$ (in press)

Cowley A., Stencel R., 1973, ApJ 184, 687

Coyne G.V., Magalhães A.M., 1977, AJ 82, 908

Daniel J., 1982, A\&A 111, 58

Espey B.R., Schulte-Ladbeck R.E., Kriss G., Hamann F., Schmid H.M., Johnson J., 1995, ApJ 454, L61

Feast M.W., Whitelock P.A., Catchpole R.M., Roberts G., Carter B.S., 1983, MNRAS 202, 951

Fernie J.D., 1985, PASP 97, 653

Fuensalida J.J., Rosa F., Fuentes F.J., 1988, A\&A 191, L13

Garcia M.R., Kenyon S.J., 1988, in The Symbiotic Phenomenon, Mikolajewska J., Friedjung M., Kenyon S.J., Viotti R. (eds.). Kluwer Academic Publishers, p. 27

Harries T.J., 1996, A\&A 315, 499

Harries T.J., Howarth I.D., 1996, A\&AS 119, 61

Hjellming R.M., van Gorkum J.H., Taylor A.R., Seaquist E.R., Padin S., Davis R.J., Bode M.F., 1986, ApJL 305, L71

Hutchings J.B., Cowley A.P., Redman R.O., 1975, ApJ 201, 404

Kenny H.T., Taylor A.R., Seaquist E.R., 1991, ApJ 366, 549

Kenyon S., 1986, The Symbiotic Stars. Cambridge University Press

Kenyon S., Webbink R.F., 1984, ApJ 279, 252

Khudyakova T.N., 1985, Sov. Astron. Lett. 11 (4), 262

Khudyakova T.N., 1988, in The Symbiotic Phenomenon, Mikolajewska J., Friedjung M., Kenyon S.J., Viotti R. (eds.). Kluwer Academic Publishers, p. 101

Liller W., 1974, ApJ 192, L89

Luthardt R., 1990, Astron. Nachr. 311, No. 2, 141

Mayall M.W., 1937, Ann. Harv. Coll. Obs. 105, 491

Meinunger L., 1981, Inf. Bull. Var. Stars, No. 2016

Merrill P.W., 1929a, PASP 41, 255

Merrill P.W., 1929b, ApJ 69, 330

Piirola V., 1973, A\&A 27, 383

Piirola V., 1983, IAU Coll. 72, 211
Piirola V., 1988a, Polarized Radiation of Circumstellar Origin, Coyne G.V. et al. (eds.). Univ. of Arizona Press, p. 433

Piirola V., 1988b, Polarized Radiation of Circumstellar Origin, Coyne G.V. et al. (eds.). Univ. of Arizona Press, p. 735

Schmid H.M., 1997, in Physical processes in Symbiotic binaries and related systems, Mikolajewska J. (ed.). Copernicous Foundation for Polish Astronomy, Warsaw, p. 21

Schmid H.M., Dumm T., Mürset U., Nussbaumer H., Schild H., Schmutz W., 1998, A\&A 329, 986

Schmid H.M., Schild H., 1994, A\&A 281, 145

Schulte-Ladbeck R.E., 1985, A\&A 142, 333

Schulte-Ladbeck R.E., Aspin C., Magalhães A.M., Schwarz H.E. (SLAMS), 1990, A\&AS 86, 227

Schulte-Ladbeck R.E., Magalhães A.M., 1987, A\&A 181, 213

Schwarz H.E., Aspin C., 1988, in The Symbiotic Phenomenon, Mikolajewska J., Friedjung M., Kenyon S.J., Viotti R. (eds.). Kluwer Academic Publishers, p. 471

Serkowski K., 1970, ApJ 160, 1083

Serkowski K., Mathewson D.S., Ford V.L., 1975, ApJ 196, 261

Shawl S.J., 1974, in Planets, Stars and nebulae studied with photopolarimetry, Arizona, UAP

Shawl S.J., 1975, AJ 80, 602

Skopal A., Djuras̃ević G., Jones A., Rovithis-Livaniou E., Rovithis P., 1997, in Physical processes in Symbiotic binaries and related systems, Mikolajewska J. (ed.). Copernicous Foundation for Polish Astronomy, Warsaw, p. 201

Slovak M.H., 1982, J. Am. Assoc. Var. Star Obs. 11, 67

Slovak M.H., Lambert D.L., 1988, in The Symbiotic Phenomenon Mikolajewska J., Friedjung M., Kenyon S.J., Viotti R. (eds.). Kluwer Academic Publishers, p. 265 Thackeray A.D., Hutchings J.B., 1974, MNRAS 167, 319

van Genderen A.M., Glass I.S., Feast M.W., 1974, MNRAS 167, 283

Webster B.L., 1966, PASP 78, 136

Webster B.L., 1973, MNRAS 164, 381 